TRANSACTIONS OF THE

AMERICAN MATHEMATICAL SOCIETY

Volume 365, Number 10, October 2013, Pages 5367-5391

S 0002-9947(2013)05809-6

Article electronically published on April 9, 2013

\title{
LAMINATIONS IN THE LANGUAGE OF LEAVES
}

\author{
ALEXANDER M. BLOKH, DEBRA MIMBS, LEX G. OVERSTEEGEN, \\ AND KIRSTEN I. S. VALKENBURG
}

This paper is dedicated to the memory of Bill Thurston

\begin{abstract}
Thurston defined invariant laminations, i.e. collections of chords of the unit circle $\mathbb{S}$ (called leaves) that are pairwise disjoint inside the open unit disk and satisfy a few dynamical properties. To be directly associated to a polynomial, a lamination has to be generated by an equivalence relation with specific properties on $\mathbb{S}$; then it is called a q-lamination. Since not all laminations are q-laminations, then from the point of view of studying polynomials the most interesting are those which are limits of q-laminations. In this paper we introduce an alternative definition of an invariant lamination, which involves only conditions on the leaves (and avoids gap invariance). The new class of laminations is slightly smaller than that defined by Thurston and is closed. We use this notion to elucidate the connection between invariant laminations and invariant equivalence relations on $\mathbb{S}$.
\end{abstract}

\section{INTRODUCTION}

Invariant laminations, introduced by Thurston in the early 1980s, are used to study the dynamics of individual polynomials and the parameter space of all polynomials, the latter in the quadratic case (an expanded version of Thurston's preprint recently appeared in Thu09]). Investigating the space of all quadratic invariant laminations played a crucial role in Thu09]. An important idea of Thurston's was, as we see it, similar to one of the main ideas of dynamics as a whole - to suggest a tool (laminations) allowing one to model the dynamics under investigation on a topologically/combinatorially nice object (in the case of [Thu09] one models polynomial dynamics on the Julia set by so-called topological polynomials, generated by laminations).

According to Thurston, a lamination $\mathcal{L}$ is a closed family of chords inside the open unit disk $\mathbb{D}$. These chords meet at most in a common endpoint and satisfy some dynamical conditions. These chords are called leaves (of the lamination), and the union of all leaves from $\mathcal{L}$ united with $\mathbb{S}$ is denoted by $\mathcal{L}^{*}$. A natural direct way to associate a lamination to a polynomial $P$ of degree $d$ with a locally connected Julia set is as follows: (1) define an equivalence relation $\sim_{P}$ on $\mathbb{S}$ by identifying

Received by the editors January 18, 2011 and, in revised form, February 14, 2012.

2010 Mathematics Subject Classification. Primary 37F20; Secondary 37F10.

Key words and phrases. Thurston lamination, complex polynomial, Julia set.

The first and third authors were supported in part by NSF-DMS-0901038 and NSF-DMS0906316. The fourth author was supported by the Netherlands Organization for Scientific Research (NWO), under grant 613.000.551; she also thanks the Department of Mathematics at the University of Alabama at Birmingham for its hospitality.

(C) 2013 American Mathematical Society Reverts to public domain 28 years from publication 
angles if their external rays land at the same point (observe that $\sim_{P}$ on $\mathbb{S}$ is $\sigma_{d^{-}}$ invariant); (2) consider the edges of convex hulls of equivalence classes and declare them to be the leaves of the corresponding lamination $\mathcal{L}_{P}$.

By Kiw04, BCO08, more advanced methods allow one to associate a lamination to some polynomials with non-locally connected Julia sets (by declaring two angles equivalent if impressions of their external rays are non-disjoint and extending this relation by transitivity). We call laminations, generated by equivalence relations similar to $\sim_{P}$ above, q-laminations. They form an important class of laminations, many of which correspond to complex polynomials with connected Julia sets. In all of these cases the lamination is found through the study of the topology of the Julia set of the polynomial.

The drawback of this approach is that it fails if the topology of the Julia set is complicated (e.g., if a quadratic polynomial has a fixed Cremer point [BO06]). Thus, even though ultimately laminations are a tool which allows one to study both individual polynomials and their parameter space, in some cases it is not obvious as to what laminations (or what equivalence relations on the circle) can be directly connected in a meaningful way to certain polynomials. Hence one needs a non-direct way of associating a lamination (or, more generally, some combinatorial structure) to a polynomial with a complicated Julia set.

A possibility here is as follows. For a polynomial $P_{c}(z)=z^{2}+c$, consider sequences of parameters $c_{i} \rightarrow c$ with $P_{c_{i}}=P_{i}$ having locally connected Julia sets and associated lamination $\mathcal{L}_{P_{i}}$. These laminations $\mathcal{L}_{P_{i}}$ (systems of chords of $\mathbb{S}$ ) may converge to another lamination (system of chords of $\mathbb{S}$ ) in the sense that the continua $\mathcal{L}_{P_{i}}^{*}$ may converge to a subcontinuum of $\mathbb{D}$ in the Hausdorff sense, and the limit continuum $\mathcal{L}^{*}$ then comes from an appropriate lamination $\mathcal{L}$. In this case the lamination $\mathcal{L}$ is called the Hausdorff limit of laminations $\mathcal{L}_{P_{i}}$; one may associate all such Hausdorff limit laminations to $c$.

Using this notion of convergence one can define the Hausdorff closures of sets of laminations. Hence the space of laminations useful for studying polynomials could be a closed set of laminations which contains the Hausdorff closure of the set of all q-laminations, but is not much larger.

To describe a candidate set of laminations we introduce a new notion of a sibling invariant lamination which is slightly more restrictive than the one given by Thurston. The new definition is given intrinsically (i.e., by only listing properties on the leaves of the lamination). We show that the family of all sibling invariant laminations is closed and contains all q-laminations. The new definition significantly simplifies the verification of the fact that a system of chords of $\mathbb{S}$ is an invariant lamination. Thurston [Thu09] introduced the class of clean laminations. We use our tools to show that clean laminations are (up to a finite modification) q-laminations. In Section 6 we apply these ideas to the degree 2 case and show that in this case all clean Thurston invariant laminations are q-laminations.

\section{Laminations: Classical definitions}

2.1. Preliminaries. Let $\mathbb{C}$ be the complex plane, $\mathbb{S} \subset \mathbb{C}$ the unit circle identified with $\mathbb{R} / \mathbb{Z}$ and let $\mathbb{D} \subset \mathbb{C}$ be the open unit disk. Define a map $\sigma_{d}: \mathbb{S} \rightarrow \mathbb{S}$ by $\sigma_{d}(z)=d z \bmod 1, d \geq 2$. By a chord in the unit disk we mean a segment of a straight line connecting two points of the unit circle. A prelamination $\mathcal{L}$ is a collection of chords in $\mathbb{D}$, called leaves, such that any two leaves of $\mathcal{L}$ meet at most 
in a point of $\mathbb{S}$. If all points of the circle are elements of $\mathcal{L}$ (seen as degenerate leaves) and $\bigcup \mathcal{L}=\mathcal{L}^{*}$ is closed in $\mathbb{C}$, then we call $\mathcal{L}$ a lamination. Hence, one obtains a lamination by closing a prelamination and adding all points of $\mathbb{S}$ viewed as degenerate leaves. If $\ell \in \mathcal{L}$ and $\ell \cap \mathbb{S}=\{a, b\}$, then we write $\ell=\overline{a b}$. We use the term "leaf" to refer to a non-degenerate leaf, and specify when a leaf may be degenerate, i.e. a point in $\mathbb{S}$.

Given a leaf $\ell=\overline{a b} \in \mathcal{L}$, let $\sigma_{d}(\ell)$ be the chord with endpoints $\sigma_{d}(a)$ and $\sigma_{d}(b)$. If $\sigma_{d}(a)=\sigma_{d}(b)$, call $\ell$ a critical leaf and $\sigma_{d}(a)$ a critical value. Let $\sigma_{d}^{*}: \mathcal{L}^{*} \rightarrow \overline{\mathbb{D}}$ be the linear extension of $\sigma_{d}$ over all the leaves in $\mathcal{L}$. It is not hard to check that $\sigma_{d}^{*}$ is continuous. Also, $\sigma_{d}$ is locally one-to-one on $\mathbb{S}$, and $\sigma_{d}^{*}$ is one-to-one on any given non-critical leaf. Note that if $\mathcal{L}$ is a lamination, then $\mathcal{L}^{*}$ is a continuum.

Definition 2.1 (Gap). A gap $G$ of a lamination $\mathcal{L}$ is the closure of a component of $\mathbb{D} \backslash \mathcal{L}^{*}$; its boundary leaves are called edges (of a gap). We also say that a leaf $\ell$ is an edge of $\ell$.

For each set $A \subset \overline{\mathbb{D}}$ we denote $A \cap \mathbb{S}$ by $\partial(A)$. If $G$ is a leaf or a gap of $\mathcal{L}$, then $G$ coincides with the convex hull of $\partial(G)$. If $G$ is a leaf or a gap of $\mathcal{L}$, we let $\sigma_{d}(G)$ be the convex hull of $\sigma_{d}(\partial(G))$. Also, by $\operatorname{Bd}(G)$ we denote the topological boundary of $G$. The topological boundary of $G$ is a Jordan curve which consists of leaves and points on $\mathbb{S}$ so that $\operatorname{Bd}(G) \cap \mathbb{S}=G \cap \mathbb{S}=\partial(G)$. A gap $G$ is called infinite if and only if $\partial(G)$ is infinite. A gap $G$ is called critical if $\left.\sigma_{d}\right|_{\partial G}$ is not one-to-one. There are two types of degenerate leaves of $\mathcal{L}^{*}$ which are not endpoints of non-degenerate leaves: (1) certain vertices of gaps, (2) points of $\mathbb{S}$, separated from other points of $\mathbb{S}$ by a sequence of leaves of $\mathcal{L}$.

2.2. q-laminations. Let $P$ be a complex polynomial with locally connected Julia set $J$. Then $J$ is connected and there exists a conformal map $\varphi: \mathbb{C}^{*} \backslash \overline{\mathbb{D}} \rightarrow \mathbb{C}^{*} \backslash K$, where $K$ is the filled-in Julia set (i.e., the complement of the unbounded component of $J$ in $\mathbb{C}$ ). One can choose $\varphi$ so that $\varphi^{\prime}(0)>0$ and $P \circ \varphi=\varphi \circ \sigma_{d}$, where $\sigma_{d}(z)=z^{d}$ and $d$ is the degree of $P$. Since $J$ is locally connected, $\varphi$ extends over the boundary $\mathbb{S}$ of $\mathbb{D}$. We also denote the extended map by $\varphi$. Define an equivalence relation $\approx_{P}$ on $\mathbb{S}$ by $x \approx_{P} y$ if and only if $\varphi(x)=\varphi(y)$. Since $J$ is the boundary of $\mathbb{C}^{*} \backslash K$, then $J$ is homeomorphic to $\mathbb{S} / \approx_{P}$. Clearly, the map $\sigma_{d}$ induces a map $f_{d}: \mathbb{S} / \approx_{P} \rightarrow \mathbb{S} / \approx_{P}$ and the maps $\left.P\right|_{J}$ and $f_{d}$ are conjugate. It is known that all equivalence classes of $\approx_{P}$ are finite. The collection of boundary edges of convex hulls of all equivalence classes of $\approx_{P}$ is a lamination denoted by $\mathcal{L}_{P}$.

Equivalence relations analogous to $\approx_{P}$ can be introduced with no reference to polynomials BL02. Let $\sim$ be an equivalence relation on $\mathbb{S}$. Equivalence classes of $\sim$ will be called $(\sim-)$ classes and will be denoted by Gothic letters. Also, given a closed set $A \subset \mathbb{C}$, let $\mathrm{CH}(A)$ denote the convex hull of the set $A$ in $\mathbb{C}$.

Definition 2.2. An equivalence relation $\sim$ is a (d-)invariant laminational equivalence relation if:

$(\mathrm{E} 1) \sim$ is closed: the graph of $\sim$ is a closed set in $\mathbb{S} \times \mathbb{S}$;

(E2) $\sim$ is unlinked: if $\mathfrak{g}_{1}$ and $\mathfrak{g}_{2}$ are distinct $\sim$-classes, then their convex hulls $\mathrm{CH}\left(\mathfrak{g}_{1}\right), \mathrm{CH}\left(\mathfrak{g}_{2}\right)$ in the unit disk $\mathbb{D}$ are disjoint,

$(\mathrm{D} 1) \sim$ is forward invariant: for a class $\mathfrak{g}$, the set $\sigma_{d}(\mathfrak{g})$ is also a class which implies that

(D2) $\sim$ is backward invariant: for a class $\mathfrak{g}$, its preimage $\sigma_{d}^{-1}(\mathfrak{g})=\{x \in \mathbb{S}:$ $\left.\sigma_{d}(x) \in \mathfrak{g}\right\}$ is a union of classes; 
(D3) for any $\sim$-class $\mathfrak{g}$ with more than two points, the map $\left.\sigma_{d}\right|_{\mathfrak{g}}: \mathfrak{g} \rightarrow \sigma_{d}(\mathfrak{g})$ is a covering map with positive orientation, i.e., for every connected component $(s, t)$ of $\mathbb{S} \backslash \mathfrak{g}$ the arc in the circle $\left(\sigma_{d}(s), \sigma_{d}(t)\right)$ is a connected component of $\mathbb{S} \backslash \sigma_{d}(\mathfrak{g})$;

(D4) all $\sim$-classes are finite.

There is an important connection between laminations and (invariant laminational) equivalence relations.

Definition 2.3. Let $\mathcal{L}$ be a lamination. Define the equivalence relation $\approx_{\mathcal{L}}$ by declaring that $x \approx_{\mathcal{L}} y$ if and only if there exists a finite concatenation of leaves of $\mathcal{L}$ joining $x$ to $y$.

Now we are ready to define q-laminations.

Definition 2.4. A lamination $\mathcal{L}$ is called a q-lamination if the equivalence relation $\approx_{\mathcal{L}}$ is an invariant laminational equivalence relation and $\mathcal{L}$ consists exactly of boundary edges of the convex hulls of all $\approx_{\mathcal{L}}$-classes together with all points of $\mathbb{S}$. If an invariant laminational equivalence relation $\sim$ is given and $\mathcal{L}$ is formed by all edges from the convex hulls of all $\sim$-classes together with all points of $\mathbb{S}$, then $\mathcal{L}$ is called the q-lamination (generated by $\sim$ ) and is denoted by $\mathcal{L}_{\sim}$. Clearly, if $\mathcal{L}$ is a q-lamination, then it is a q-lamination generated by $\approx_{\mathcal{L}}$.

Let $\sim$ be a laminational equivalence relation and $p: \mathbb{S} \rightarrow J_{\sim}=\mathbb{S} / \sim$ be the quotient map of $\mathbb{S}$ onto its quotient space $J_{\sim}$, and let $f_{\sim}: J_{\sim} \rightarrow J_{\sim}$ be the map induced by $\sigma_{d}$. We call $J_{\sim}$ a topological Julia set and the induced map $f_{\sim}$ a topological polynomial. It is easy to see from Definition 2.2 that leaves of $\mathcal{L}_{\sim}$ map to leaves of $\mathcal{L}_{\sim}$ under $\sigma_{d}$; moreover, the map $\sigma_{d}$ acting on leaves and gaps of $\mathcal{L}_{\sim}$ also has other more specific properties analogous to (D1) - (D4) above. This leads to the abstract notion of an invariant lamination Thu09 that allows for laminations which are not directly associated to a laminational equivalence relation and, hence, do not correspond (directly) to a polynomial.

\subsection{Invariant laminations due to Thurston.}

Definition 2.5 (Monotone map). Let $X, Y$ be topological spaces and $f: X \rightarrow Y$ be continuous. Then $f$ is said to be monotone if $f^{-1}(y)$ is connected for each $y \in Y$. It is known that if $f$ is monotone and $X$ is a continuum, then $f^{-1}(Z)$ is connected for every connected $Z \subset f(X)$.

Definition 2.6 is due to Thurston [Thu09].

Definition 2.6 (Thurston invariant lamination Thu09]). A lamination $\mathcal{L}$ is Thurston d-invariant if it satisfies the following conditions.

(1) Forward $d$-invariance: for any leaf $\ell=\overline{p q} \in \mathcal{L}$, either $\sigma_{d}(p)=\sigma_{d}(q)$ or $\overline{\sigma_{d}(p) \sigma_{d}(q)}=\sigma_{d}(\ell) \in \mathcal{L}$.

(2) Backward invariance: for any leaf $\overline{p q} \in \mathcal{L}$, there exists a collection of $d$ disjoint leaves in $\mathcal{L}$ (this collection of leaves may not be unique), each joining a preimage of $p$ to a preimage of $q$.

(3) Gap invariance: For any gap $G$, the convex hull $H$ of $\sigma_{d}(G \cap \mathbb{S})$ is a gap, a leaf, or a single point (of $\mathbb{S}$ ).

If $H$ is a gap, $\left.\sigma_{d}^{*}\right|_{\mathrm{Bd}(G)}: \operatorname{Bd}(G) \rightarrow \operatorname{Bd}(H)$ maps as the composition of a monotone map and a covering map to the boundary of the image gap, with positive orientation (the image of a point moving clockwise around $\operatorname{Bd}(G)$ must move clockwise around the image $\operatorname{Bd}(H)$ of $G)$. 
By Lemma 2.1 of [BL02, any q-lamination is Thurston invariant (the only part which is not immediate deals with the gap-invariance of infinite gaps of any qlamination).

\section{Sibling INVARIANT LAMINATIONS}

3.1. An alternative definition. Note that in Definition 3.1 we do not require the invariance of gaps.

Definition 3.1 (Sibling $d$-invariant lamination Mim10). A (pre)lamination $\mathcal{L}$ is sibling d-invariant if:

(1) for each $\ell \in \mathcal{L}$ either $\sigma_{d}(\ell) \in \mathcal{L}$ or $\sigma_{d}(\ell)$ is a point in $\mathbb{S}$,

(2) for each $\ell \in \mathcal{L}$ there exists a leaf $\ell^{\prime} \in \mathcal{L}$ such that $\sigma_{d}\left(\ell^{\prime}\right)=\ell$,

(3) for each $\ell \in \mathcal{L}$ such that $\sigma_{d}(\ell)$ is a non-degenerate leaf, there exist $\mathbf{d}$ disjoint leaves $\ell_{1}, \ldots, \ell_{d}$ in $\mathcal{L}$ such that $\ell=\ell_{1}$ and $\sigma_{d}\left(\ell_{i}\right)=\sigma_{d}(\ell)$ for all $i$.

Let us make a few remarks. For a continuum/point $K \subset \mathcal{L}^{*}$ which maps one-toone onto $\sigma_{d}^{*}(K)$, we call a continuum/point $T \subset \mathcal{L}^{*}$ a sibling (of $K$ ) if $K \cap T=\emptyset$ and $T$ maps onto $\sigma_{d}^{*}(K)$ in a one-to-one fashion (thus, siblings are homeomorphic and disjoint). E.g., the leaves $\ell_{2}, \ldots, \ell_{d}$ from Definition 3.1 are siblings of $\ell$. The collection $\left\{\ell, \ell_{2}, \ldots, \ell_{d}\right\}$ of leaves from Definition 3.1 is called a full sibling collection (of $\ell$ ). For a continuum/point $K \subset \mathcal{L}^{*}$ which maps one-to-one onto $\sigma_{d}^{*}(K)$, a collection of $d$ sets made up of $K$ and its pairwise disjoint siblings is called a full sibling collection (of $K$ ). Often we talk about siblings without assuming the existence of a full sibling collection (e.g., in the context of Thurston $d$-invariant laminations).

Let $\mathcal{L}$ be a sibling $d$-invariant lamination. By Definition 3.1 (1) we see that Definition 2.6 (1) is satisfied. Let $\ell \in \mathcal{L}$ be a leaf. By Definition 3.1 (2) and (3) there are $d$ pairwise disjoint leaves of $\mathcal{L}$ mapped onto $\ell$; hence, Definition 2.6 (2) is satisfied. Thus both sibling $d$-invariant laminations and Thurston $d$-invariant laminations satisfy conditions (1) and (2) of Definition 2.6, i.e. are forward $d$ invariant and backward d-invariant. These conditions deal with leaves and in that respect are intrinsic to $\mathcal{L}$, defined as a collection of leaves. However, having these conditions is not enough to define a meaningful dynamic collection of leaves; there are examples of laminations satisfying conditions (1)-(2) of Definition 2.6] which are not gap invariant. Hence one needs to add an extra condition to forward and backward invariance.

In Definition 2.6 one deals with gaps, i.e. closures of components of the complement $\mathbb{D} \backslash \mathcal{L}^{*}$. This is a direct way to ensure that $\sigma_{d}^{*}$ has a nice extension over the plane. A drawback of this approach is that while $\mathcal{L}$ otherwise is defined as a family of chords of $\mathbb{D}$ (leaves), in gap invariance we talk about other objects (gaps). One can argue that gap invariance of $\mathcal{L}$ under $\sigma_{d}$ is not sufficiently intrinsic since $\mathcal{L}$ is defined as a collection of leaves. As a consequence it is often more cumbersome to verify gap invariance. This justifies the search for a similar definition of an invariant lamination which deals only with leaves. Above we propose the notion of a sibling (d)-invariant lamination.

3.2. Sibling invariant laminations are gap invariant. We show that any sibling $d$-invariant lamination is Thurston $d$-invariant. Some complications below are caused by the fact that we do not yet know that the lamination is gap invariant. 
E.g., extending the map $\sigma^{*}$ over $\mathbb{D}$ to a suitably nice map (i.e., the composition of a monotone and open map) is impossible if the lamination is not gap invariant.

Theorem 3.2. Suppose that $G$ is a gap of a sibling d-invariant lamination $\mathcal{L}$. Then either

(1) $\sigma_{d}(G)$ is a point in $\mathbb{S}$ or a leaf of $\mathcal{L}$ or

(2) there is a gap $H$ of $\mathcal{L}$ such that $\sigma_{d}(G)=H$, and the map $\left.\sigma_{d}^{*}\right|_{\operatorname{Bd}(G)}$ : $\mathrm{Bd}(G) \rightarrow \operatorname{Bd}(H)$ is the positively oriented composition of a monotone map $m: \operatorname{Bd}(G) \rightarrow S$, where $S$ is a simple closed curve, and a covering map $g: S \rightarrow \operatorname{Bd}(H)$.

Thus, any sibling d-invariant lamination is Thurston d-invariant.

To prove Theorem 3.2 we prove a few lemmas. Given a point $x$, call any point $\hat{x} \in \mathbb{S}$ with $\sigma_{d}(\hat{x})=x$ an $x$-point. If a lamination is given, by an $\hat{a} \hat{b}$-leaf, we mean a leaf that maps to $\overline{a b}$. The word "chord" is used in lieu of "leaf" in reference to a chord of $\mathbb{D}$ which may not be a leaf of $\mathcal{L}$. By $[a, b]_{\mathbb{S}}, a, b \in \mathbb{S}$ we mean the closed arc of $\mathbb{S}$ from $a$ to $b$, and by $(a, b)_{\mathbb{S}}$ we mean the open arc of $\mathbb{S}$ from $a$ to $b$. The direction of the arc, clockwise (negative) or counterclockwise (positive), will be clear from the context; also, sometimes we simply write $[a, b],(a, b)$, etc. By $<$ we denote the positive (circular) order on $\mathbb{S}$. If we say that points are ordered on $\mathbb{S}$ we mean that they are either positively or negatively ordered. Proposition 3.3 is left to the reader. Observe that in Proposition 3.3 we do not assume the existence of a lamination.

Proposition 3.3. Suppose that $a_{1}<b_{1}<a_{2}<b_{2}<\cdots<a_{n}<b_{n}$ are $2 n$ points in the circle. Then for any point $a_{i}$ and $b_{j}$ either component of $\mathbb{S} \backslash\left\{a_{i}, b_{j}\right\}$ contains the same number of a-points and b-points. Thus, if $\hat{a}, \hat{b} \in \mathbb{S}$ are such that $a=\sigma_{d}(\hat{a}) \neq \sigma_{d}(\hat{b})=b$, then either component of $\mathbb{S} \backslash\{\hat{a}, \hat{b}\}$ contains the same number of a-points and b-points.

Since 2-invariant laminations are invariant under the rotation by $\frac{1}{2}$, then, given a 2-invariant lamination we see that its siblings are rotations of each other. Even though this is not typically true for laminations of higher degree (see Figure 11), Lemma 3.6 states that sibling leaves must connect in the same order. To state it we need Definition 3.4 .

Definition 3.4. Consider two sets $A, C \subset \mathbb{S}$ such that $\sigma_{d}(A)=C$ and $\left.\sigma_{d}\right|_{A}$ is oneto-one. Then $A$ and $C$ are said to have the same (circular) orientation if for any three points $x, y, z \in A$, their images $\sigma_{d}(x), \sigma_{d}(y), \sigma_{d}(z)$ have the same orientation as $x, y, z$. In this case we say that $\sigma_{d}$ preserves orientation on $A$. If there is another set $B$ satisfying the same properties as $A$ above (so that $C$ is a one-to-one $\sigma_{d}$-image of $B$, etc.), we say that $A$ and $B$ have the same orientation. Now, if $\left.\sigma_{d}\right|_{A}$ is not oneto-one and for any three points $x, y, z \in A$ their images $\sigma_{d}(x), \sigma_{d}(y), \sigma_{d}(z)$ either have the same orientation as $x, y, z$ or some of them coincide, then we say that $\left.\sigma_{d}\right|_{A}$ weakly preserves orientation.

If $\sigma_{d}(A)=\sigma_{d}(B)=C$ and $\sigma_{d}$ preserves orientation of $A$ and $B$, then, as we walk along the circle in the positive direction from a point $u \in A$, its sibling $u^{\prime} \in B$, and its image $\sigma_{d}(u)$, we will meet points of $A$, their siblings in $B$, and their images in $C$ in the same order.

Any two two-point sets which are not critical and map to the same set have the same orientation; this is not necessarily true for sets with more points. The fact 


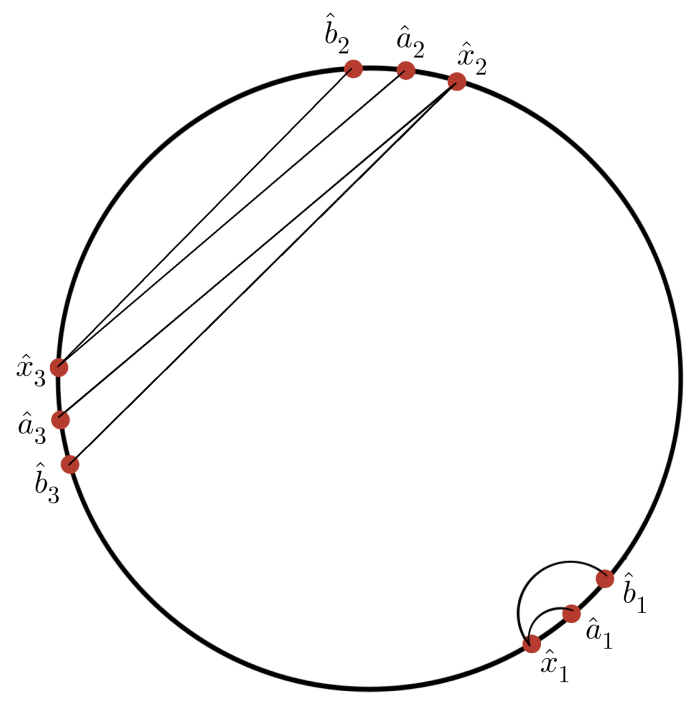

Figure 1. Sibling "arcs"

This is an example of sibling "arcs" under $\sigma_{3}$. Notice that while the arcs connect points in different "patches" and are not found by rigid rotation, the manner in which the endpoints connect preserves order.

that sets have the same orientation sometimes implies "structural" conclusions. For a set $A \subset \mathcal{L}^{*}$ we write $A \sqsubset \mathcal{L}^{*}$ if $A \cap \mathbb{S}$ is zero dimensional.

Definition 3.5. A triod is a homeomorphic image of the simple triod $\tau$ (the union of three arcs which share a common endpoint). Denote by $B(T)$ the union of the endpoints and the vertex of a triod $T$. In what follows we always consider triods $T \subset \mathbb{D}$ with $B(T) \subset \mathbb{S}$. The edge of $T$, which separates (inside $\overline{\mathbb{D}}$ ) the endpoints of $T$ not belonging to it, is called the central edge of $T$ while the other edges of $T$ are said to be sides of $T$. Similarly, if $A$ is the union (the concatenation) of two leaves $\overline{a v} \cup \overline{v b}$, we set $B(A)=\{a, v, b\}$.

To avoid ambiguity we call a subarc of $\mathbb{S}$ a circle arc. By an arc in $\mathcal{L}^{*}$ we mean a topological arc (a one-to-one image of an interval). Given a set $A \subset \mathcal{L}^{*}$ we sometimes need to consider a topological arc in $A$ with endpoints $x, y$ (it will always be clear which arc we actually consider); such an arc will be denoted by $[x, y]_{A}$. Thus, $[a, b]_{\mathbb{S}}$ is always a circle arc. By default arcs $[a, b],(a, b)$, etc. are circle arcs.

Lemma 3.6. (1) Let $x_{1}<a_{1}<b_{1}<x_{2}<\cdots<x_{n}<a_{n}<b_{n}<x_{1}$ be $3 n$ points in $\mathbb{S}$. If for each $i$ there exists $r(i), m(i) \in\{1, \ldots, n\}$ such that arcs $A_{i}=\overline{x_{i} a_{r(i)}} \cup \overline{x_{i} b_{m(i)}}$ are pairwise disjoint $(1 \leq i \leq n)$, then $x_{i}<a_{r(i)}<b_{m(i)}$ for each $i$.

(2) Let $x_{1}<a_{1}<b_{1}<c_{1}<x_{2}<\cdots<x_{n}<a_{n}<b_{n}<c_{n}<x_{1}$ be $4 n$ points in $\mathbb{S}$. If for each $i$ there exist $r(i), m(i), l(i) \in\{1, \ldots, n\}$ such that triods $T_{i}=\overline{x_{i} a_{r(i)}} \cup \overline{x_{i} b_{m(i)}} \cup \overline{x_{i} c_{l(i)}}$ are pairwise disjoint $(1 \leq i \leq d)$, then $x_{i}<a_{r(i)}<$ $b_{m(i)}<c_{l(i)}$ for each $i$. 
Proof. (1) Let $x_{1}<b_{m(1)}<a_{r(1)}$. Then the arc $\left(x_{1}, b_{m(1)}\right)$ contains $m(1)-1$ points $x_{2}, \ldots, x_{m(1)}, m(1)-1$ points $b_{1}, \ldots, b_{m(1)-1}$, but $m(1)$ points $a_{1}, \ldots, a_{m(1)}$. This contradicts the existence of sets $A_{j}$.

(2) Follows from (1) applied to parts of the triods $T_{i}$.

We will mostly apply the following corollary of Lemma 3.6

Corollary 3.7. Let $\mathcal{L}$ be a sibling d-invariant lamination and $T \sqsubset \mathcal{L}^{*}$ be an arc consisting of two leaves with a common endpoint $v$ or a triod consisting of three leaves with a common endpoint $v$. Suppose that $S \sqsubset \mathcal{L}^{*}$ is an arc (triod) such that $\sigma_{d}^{*}(S)=T$ and $\left.\sigma_{d}^{*}\right|_{S}$ is one-to-one. Then the orientation of the sets $B(T)$ and $B(S)$ is the same.

Proof. The set of all preimages of points of $B(T)$ consists of $d$ triples (if $T$ is an arc) or quadruples (if $T$ is a triod) of points denoted by $B_{1}, \ldots, B_{d}$ and such that (1) each $B_{i}$ is contained in a circle arc $J_{i}$ so that these arcs are disjoint and of the same length less than $\frac{1}{d}$, (2) the circular order of points in $B_{i}$ is the same as the circular order of $\sigma_{d}$-images of these points.

Denote by $v$ the point from $B(T)$ which is either the vertex of $T$ (if $T$ is a triod) or a point from $B(T)$ which is not an endpoint of $T$ (if $T$ is an arc). Take the leaves which comprise $T$ (two leaves if $T$ is an arc and three leaves if $T$ is a triod). Consider the corresponding leaves comprising $S$. Each leaf of $S$ gives rise to its full sibling collection (here we use the fact that $\mathcal{L}$ is sibling invariant). Then leaves from those collections "grow" out of points $v_{1}, \ldots, v_{d}$ which are preimages of $v$. This gives rise to $d$ unlinked sets $S_{1}, \ldots, S_{d}$, where $S_{i}$ is a union of two (three) leaves growing out of $v_{i}$ (indeed, no leaf of $S_{i}$ can coincide with a leaf of $S_{j}$ where $i \neq j$, while distinct leaves must be disjoint by the properties of laminations). Moreover, we may assume that $S_{1}=S$. It now follows from Lemma 3.6 and the above paragraph that all the sets $B\left(S_{i}\right)$ have the same orientation coinciding with the orientation of the set of their $\sigma_{d}$-images, i.e. the set $B(T)$.

Corollary 3.7 shows that all pullbacks of certain sets have the same orientation as the sets themselves. However it also allows us to study images of some sets. Indeed, by Corollary 3.7, if $S \sqsubset \mathcal{L}^{*}$ is a triod mapped by $\sigma_{d}^{*}$ one-to-one to $T$, then the central edge of $S$ maps into the central edge of $T$.

Lemma 3.6 and Corollary 3.7 are useful in comparing the orientation of arcs (triods) and their images in the absence of critical leaves. In the case when there are critical leaves in the arcs and triods we need additional lemmas. We will need the following terminology.

By a preimage collection (of a chord $\overline{a b}$ ) we mean a collection $A$ of several pairwise disjoint chords with the same non-degenerate image-chord $\overline{a b}$; here we do not necessarily assume the existence of a lamination. However, if we deal with a lamination $\mathcal{L}$, then we always assume that preimage collections consist of leaves of $\mathcal{L}$. If there are $d$ disjoint chords in $A$ we call it full. If $X$ is a preimage collection of a chord $\overline{a b}$, we denote the endpoints of chords of $X$ by the same letters as for the endpoints of their images but with a hat and distinct subscripts, and call them correspondingly ( $a$-points, $b$-points, etc.). Given two sibling chords $\ell^{\prime}$ and $\ell^{\prime \prime}$ with non-degenerate image, we say that they are ordered if no two sibling endpoints of these chords are adjacent on $\mathbb{S}$. Thus, if $\overline{\hat{a}_{1} \hat{b}_{1}}, \overline{\hat{a}_{2} \hat{b}_{2}}$ are two sibling chords, then they are ordered if either $\hat{a}_{1}<\hat{b}_{1}<\hat{a}_{2}<\hat{b}_{2}$ or $\hat{a}_{1}<\hat{b}_{2}<\hat{a}_{2}<\hat{b}_{1}$ (equivalently, if $\overline{\hat{a}_{1} \hat{a}_{2}}$ 
separates $\overline{\hat{a}_{1} \hat{b}_{1}} \backslash\left\{\hat{a}_{1}\right\}$ from $\overline{\hat{a}_{2} \hat{b}_{2}} \backslash\left\{\hat{a}_{2}\right\}$ in $\left.\mathbb{D}\right)$. Finally, recall that $\partial(X)$ is the union of all endpoints of chords from $X$.

Lemma 3.8. Let $X$ be a full preimage collection of a chord $\overline{a b}$ and let $\ell_{1}=\overline{\hat{a}_{1} \hat{b}_{1}}$, $\ell_{2}=\overline{\hat{a}_{2} \hat{b}_{2}}$ be two chords from $X$. Then the number of chords from $X$ crossing the chord $\overline{\hat{a}_{1} \hat{a}_{2}}$ inside $\mathbb{D}$ is even if and only if $\ell_{1}$ and $\ell_{2}$ are ordered. In particular, suppose that one of the following holds:

(1) there exists a concatenation $Q$ of chords connecting $\hat{a}_{1}$ and $\hat{a}_{2}$ and disjoint with chords of $X$ except for the points $\hat{a}_{1}, \hat{a}_{2}$;

(2) there exists a concatenation $Q$ of critical chords connecting $\hat{a}_{1}$ and $\hat{a}_{2}$ and such that all their endpoints distinct from $\hat{a}_{1}$ and $\hat{a}_{2}$ belong to the component of $\mathbb{S} \backslash\left\{\hat{a}_{1}, \hat{a}_{2}\right\}$ not containing $\hat{b}_{1}$.

Then $\ell_{1}$ and $\ell_{2}$ are ordered.

Proof. See Figure 2, First let us show that if, say, $\hat{a}_{1}<\hat{b}_{1}<\hat{a}_{2}<\hat{b}_{2}$, then the number of chords from $X$ crossing the chord $\overline{\hat{a}}_{1} \hat{a}_{2}$ inside $\mathbb{D}$ is even. Indeed, by Proposition 3.3 there are, say, $k a$-points and $k b$-points in $\left(\hat{b}_{1}, \hat{a}_{2}\right)$. Suppose that among chords of $X$ there are $m$ chords with both endpoints in $\left(\hat{b}_{1}, \hat{a}_{2}\right)$. Then there are $2 k-2 m a$ - and $b$-points in $\left(\hat{b}_{1}, \hat{a}_{2}\right)$ which are exactly all the endpoints of chords from $X$ which cross $\overline{\hat{a}_{1} \hat{a}_{2}}$. inside $\mathbb{D}$. This implies that the number of chords from $X$ crossing the chord $\bar{a}_{1} \hat{a}_{2}$ inside $\mathbb{D}$ is even.

On the other hand, suppose that the number of chords from $X$ crossing the chord $\bar{a}_{1} \hat{a}_{2}$ inside $\mathbb{D}$ is even. As before, for definiteness assume that $\hat{a}_{1}<\hat{b}_{1}<\hat{b}_{2}<\hat{a}_{1}$. For any $Z \subset X$ consider a function $\varphi(I, Z)$ of an $\operatorname{arc} I \subset \mathbb{S}$, defined as the difference between the number of $a$-points in $Z \cap I$ and the number of $b$-points in $Z \cap I$ taken modulo 2. Clearly, for some $k$ the $\operatorname{arc}\left(\hat{b}_{1}, \hat{b}_{2}\right)$ contains $k b$-points and $k+1 a$-points; similarly, for some $l$ the $\operatorname{arc}\left(\hat{a}_{2}, \hat{a}_{1}\right)$ contains $l a$-points and $l+1 b$-points. Hence, $\varphi\left(\left(\hat{b}_{1}, \hat{b}_{2}\right), X\right)=\varphi\left(\left(\hat{a}_{2}, \hat{a}_{1}\right), X\right)=1$.

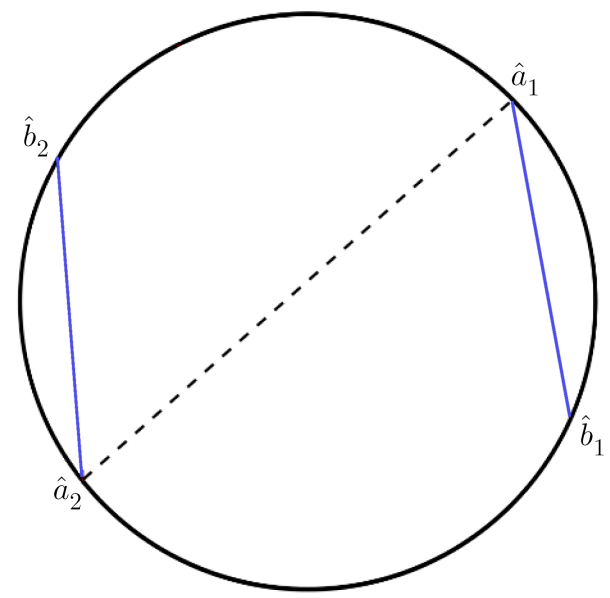

FiguRE 2. Siblings and critical leaves

Remove all the chords from $X$ connecting the arcs $\left(\hat{b}_{1}, \hat{b}_{2}\right)$ and $\left(\hat{a}_{2}, \hat{a}_{1}\right)$ to get a new set of chords $Y$. As we remove one such chord, we change the value of $\varphi$ 
on $\left(\hat{b}_{1}, \hat{b}_{2}\right)$ by 1 , and we change the value of $\varphi$ on $\left(\hat{a}_{2}, \hat{a}_{1}\right)$ by 1 as well. By the assumption, to get the set $Y$ we need to remove an even number of chords. Thus, we see that $\varphi\left(\left(\hat{b}_{1}, \hat{b}_{2}\right), Y\right)=\varphi\left(\left(\hat{a}_{2}, \hat{a}_{1}\right), Y\right)=1$. However, the remaining points of $\partial(Y) \cap\left(\hat{b}_{1}, \hat{b}_{2}\right)$ are endpoints of a certain number of chords from $X$, and hence there must be an equal number of $a$-points and $b$-points among them, a contradiction.

Let us prove the remaining claims of the lemma. In case (1) there exists a concatenation $Q$ of chords connecting $\hat{a}_{1}$ to $\hat{a}_{2}$ so that $Q \cap X=\left\{\hat{a}_{1}, \hat{a}_{2}\right\}$; then no chord of $X$ can cross the chord $\overline{\hat{a}_{1} \hat{a}_{2}}$ inside $\mathbb{D}$. Hence by the first part $\ell_{1}$ and $\ell_{2}$ are ordered. Now, consider case (2). We may assume that there is a concatenation of critical chords $\overline{\hat{a}_{1} \hat{a}^{2}}, \overline{\hat{a}^{2} \hat{a}^{3}}, \ldots, \overline{\hat{a}^{i} \hat{a}_{2}}$ such that all points $\hat{a}^{2}, \ldots, \hat{a}^{i}$ belong to the $\operatorname{arc}\left(\hat{a}_{1}, \hat{a}_{2}\right)$ while $\hat{b}_{1} \in\left(\hat{a}_{2}, \hat{a}_{1}\right)$. Then, by the first part, the chord from $X$ with an endpoint $\hat{a}^{2}$ has the other endpoint $\hat{b}^{2}$ in $\left(\hat{a}_{1}, \hat{a}^{2}\right)$. Repeating this observation, we will finally see that $\hat{b}_{2} \in\left(\hat{a}^{i}, \hat{a}_{2}\right)$, as desired.

To prove further lemmas we need more definitions.

Definition 3.9. Let $I \sqsubset \mathcal{L}^{*}$ be an arc (the image of a homeomorphism $\varphi:[0,1] \rightarrow$ $I)$. We call $I$ a monotone arc if its endpoints $\varphi(0), \varphi(1)$ belong to $\mathbb{S}$ and there is a circle arc $T=[\varphi(0), \varphi(1)]_{\mathbb{S}}$ which contains $\partial(I)$ (this implies that the map $\left.\varphi^{-1}\right|_{\partial(I)}$ is monotone with respect to the circular order on $T$ ). Likewise, a triod $T \sqsubset \mathcal{L}^{*}$ is monotone if all its edges are monotone arcs.

As an example of a monotone arc one can consider a single leaf of $\mathcal{L}$ or a subarc of the boundary of a gap of $\mathcal{L}$. It turns out that in the case of sibling invariant laminations the map preserves local orientation on monotone arcs. More precisely, let $I \sqsubset \mathcal{L}^{*}$ be a monotone arc. Some leaves (finitely many) in $I$ may be critical.

Definition 3.10. Let $I \sqsubset \mathcal{L}^{*}$ be a monotone arc with endpoints $u$ and $v$ so that $\partial(I) \subset[u, v]$. Define the natural order $<_{I}$ on $\partial(I)$ induced by the positive order on the circle arc $[u, v]$. We say that $\sigma_{d}^{*}$ preserves local orientation on $I$ if for any point $b \in \partial(I)$ there exists a closed (including the endpoints) neighborhood $U$ of $b$ in the $<_{I}$-order topology on $\partial(I)$ such that either $\left.\sigma_{d}\right|_{U}$ weakly preserves orientation or $U$ consists of finitely many points and the corresponding concatenation of leaves maps by $\sigma_{d}^{*}$ to one leaf.

Lemma 3.11. If $\mathcal{L}$ is a sibling invariant lamination, then $\sigma_{d}^{*}$ preserves local orientation on any monotone arc.

Proof. Let $I \sqsubset \mathcal{L}^{*}$ be a monotone arc with endpoints $u$ and $v$ so that $\partial(I) \subset[u, v]$; denote by $<$ the positive order on $[u, v]$. Let $\mathcal{I}$ be the set $\partial(I)$ considered as a topological space endowed with $<_{I}$-order topology. Denote by $[x, y]_{\mathcal{I}}$ a closed interval in $\mathcal{I}$ with endpoints $x<_{I} y$. If $b \in \mathcal{I}$ while $\overline{a b} \subset I$ and $\overline{b c} \subset I$ are noncritical leaves, then we can choose $U=[a, b]_{\mathcal{I}}$. Lemma 3.8 implies that either $\sigma_{d}$ maps $\overline{a b c}$ to one leaf or $\sigma_{d}$ preserves orientation on $[a, b]_{\mathcal{I}}$. Suppose that there is a concatenation of leaves $\overline{a b_{1} \cdots b_{k} c} \subset I$ such that $\overline{b_{i} b_{i+1}}$ are critical leaves, $i=1, \ldots, k-1$. By Lemma 3.8 there exists a leaf $\overline{a^{\prime} b_{k}}$ which is a sibling of $\overline{a b_{1}}$ with $b_{k-1}<a^{\prime}<b_{k}$. By Lemma 3.8 either $\sigma_{d}$ preserves orientation on $\overline{a^{\prime} b_{k} c}$ or $\sigma_{d}$ maps $\overline{a^{\prime} b_{k} c}$ to a leaf. Thus, for each point $b_{i}$ we can choose $U=[a, c]_{\mathcal{I}}$.

Let $b \in \partial(B)$. Now consider cases when either $b$ is approached on at least one side by points of $\partial(I)$ or $b$ belongs to a maximal concatenation $Y$ of critical leaves in $I$ with at least one endpoint of $Y$ approached by points of $\partial(I)$. In these 
cases the arguments are easier because now we can rely upon the fact that $\left.\sigma_{d}\right|_{\mathbb{S}}$ preserves local orientation (e.g., this immediately implies our claim in the case when $b$ is non-isolated in $\partial(I)$ on either side) and because we can choose a small semi-neighborhood $V$ of $b$ on the side from which $b$ is non-isolated in $\partial(I)$ so that if there is, say, a non-critical leaf $\overline{a b} \subset I$ coming out of $b$ on the side other than $V$, the map still preserves orientation on $(V \cap \partial(I)) \cup\{a, b\}$. In the case when there is a concatenation of critical leaves containing $b$, an obvious modification of these arguments (compare with the first paragraph of the proof) yields the desired result.

We are ready to prove the following lemma.

Lemma 3.12. Let $\mathcal{L}$ be a sibling d-invariant lamination. Suppose that $\sigma_{d}^{*}$ monotonically maps a monotone arc $I$ onto the union of the two sides of a monotone triod $T$ with vertex $v$ whose central edge is a leaf $\overline{v m}$. Then there exists $\hat{v} \in \partial(I)$ such that $\sigma_{d}(\hat{v})=v$ and there exists a leaf $Q=\overline{\hat{v} \hat{m}}$ such that $I \cup Q$ is a monotone triod with vertex $u$ and central edge $Q$.

Proof. Denote the endpoints of $T$ distinct from $m$ by $a$ and $b$. Then the endpoints of $I$ map to $a$ and $b$; denote them $\hat{a}$ and $\hat{b}$, respectively. For the sake of definiteness assume that $v \in(a, b)$. Consider $\left.\sigma_{d}\right|_{(\hat{b}, \hat{a})}$. Clearly, as we move from $\hat{b}$ to $\hat{a}$ we first encounter several semi-open subarcs of $(\hat{b}, \hat{a})$ which wrap around the circle in a one-to-one fashion. Then the last arc which we encounter connects a $b$-point with an $a$-point (namely, with $\hat{a}$ ) and maps onto $[b, a]$ in a one-to-one fashion. Hence there is one more $m$-point in $(\hat{b}, \hat{a})$ than $v$-points in $(\hat{b}, \hat{a})$. This implies that one $m$-point belonging to $(\hat{b}, \hat{a})$ (denote it by $\hat{m})$ must be connected with a leaf to a $v$-point belonging to $(\hat{a}, \hat{b})$ (denote it by $\hat{v}$ ). This completes the proof.

By a polygon we mean a finite convex polygon. In what follows, by a collapsing polygon we mean a polygon $P$ with edges which are chords of $\mathbb{D}$ such that their images are the same non-degenerate chord (thus as we walk along the edges of $P$, their $\sigma_{d}$-images walk back and forth along the same non-degenerate chord). When we say that $Q$ is a collapsing polygon of a lamination $\mathcal{L}$, we mean that all edges of $Q$ are leaves of $\mathcal{L}$; we also say that $\mathcal{L}$ contains a collapsing polygon $Q$. However, this does not necessarily imply that $Q$ is a gap of $\mathcal{L}$, as $Q$ might be further subdivided by leaves of $\mathcal{L}$ inside $Q$.

We often deal with concatenations of leaves, i.e. finite collections of pairwise distinct leaves which, when united, form a topological arc in $\mathbb{D}$. The concatenation of leaves $\ell_{1}, \ldots, \ell_{k}$ is denoted $\ell_{1} \cdots \ell_{k}$. If the leaves are given by their endpoints $x_{1}, \ldots, x_{k}$, we denote the concatenation by $\overline{x_{1} \cdots x_{k}}$. We do not assume that points $x_{1}, \ldots, x_{k}$ are ordered on the circle; however, if they are, we call $\overline{x_{1} \cdots x_{k}}$ an ordered concatenation.

Lemma 3.13. Suppose that $\mathcal{L}$ is a sibling d-invariant lamination which contains two distinct leaves $\ell_{0}=\overline{v x}$ and $\ell_{1}=\overline{v y}$ such that $\sigma_{d}\left(\ell_{0}\right)=\sigma_{d}\left(\ell_{1}\right)=\ell$ is a nondegenerate leaf. Then $\mathcal{L}$ contains a collapsing polygon $P$ with edges $\ell_{0}$ and $\ell_{1}$ such that $\sigma_{d}(P)=\ell$; also, it contains a critical gap $G \subset P$ with vertex $v$ such that $\sigma_{d}(G)=\ell$.

Proof. First assume that $x<v<y$ and that there are no leaves $\ell^{\prime}=\overline{v z} \in \mathcal{L}$ with $y<z<x$ and $\sigma_{d}\left(\ell^{\prime}\right)=\ell$. Since $\mathcal{L}$ is a sibling $d$-invariant lamination, we can 
choose a full sibling collection $A_{0} \subset \mathcal{L}$ of $\ell_{0}$. By Lemma 3.8 there exists $u_{1} \in(y, x)$ such that $\overline{y u_{1}} \in A_{0}$ and $\ell_{0}$ are siblings (we apply Lemma 3.8 with $x$ and $y$ playing the roles of $\hat{a}_{1}$ and $\hat{a}_{2}$ ). Similarly, there exists a full sibling collection $A_{1} \subset \mathcal{L}$ of $\ell_{1}$ and a point $u_{0} \in(y, x)$ such that $\overline{u_{0} x} \in A_{1}$ and $\ell_{1}$ are siblings. Since $\overline{y u_{1}}$ and $\overline{u_{0} x}$ are disjoint inside $\mathbb{D}$, then $y<u_{1} \leq u_{0}<x$.

Consider all possible choices of points $u_{0}, u_{1}$ so that the above properties hold: $\overline{y u_{1}}$ and $\ell_{0}$ are siblings, $\overline{u_{0} x}$ and $\ell_{1}$ are siblings, and $y<u_{1} \leq u_{0}<x$. Observe that now we do not require that $u_{0}$ or $u_{1}$ be obtained as endpoints of siblings of $\ell_{0}$ or $\ell_{1}$ coming from full sibling collections, but the existence of such collections shows that the set of the choices is non-empty (see the first paragraph). Choose $u_{0}, u_{1}$ so that the arc $\left[u_{1}, u_{0}\right]$ is the shortest.

If $u_{0}=u_{1}=u$, then we obtain a collapsing polygon $P=\mathrm{CH}(v y u x)$. Suppose that $u_{0} \neq u_{1}$. Then by the construction and by the choice of $u_{0}$ and $u_{1}$ no leaf of $\mathcal{L}$ which maps onto $\ell$ can cross the chords $\overline{v u_{0}}, \overline{v u_{1}}$. By Lemma 3.8 applied to $A_{0}$ and $\ell_{0} \in A_{0}$, and because of the location of the points found so far, there exists a sibling $\overline{w_{0} u_{0}} \in A_{0}$ of $\ell_{0}$ with $w_{0} \in\left(u_{1}, u_{0}\right)$. Similarly, there exists a sibling $\overline{u_{1} w_{1}} \in A_{1}$ of $\ell_{1}$ with $w_{1} \in\left(u_{1}, u_{0}\right)$. Since leaves $\overline{w_{0} u_{0}}$ and $\overline{u_{1} w_{1}}$ do not intersect inside $\mathbb{D}$, we see that $u_{1}<w_{1} \leq w_{0}<u_{0}$. Similar to what we did before, we can choose $w_{1}$ and $w_{0}$ so that $\overline{w_{0} u_{0}}$ is a sibling of $\ell_{0}, \overline{w_{1} u_{1}}$ is a sibling of $\ell_{1}$, and the $\operatorname{arc}\left(w_{1} w_{0}\right)$ is the shortest possible. We can continue in this manner and obtain a collapsing polygon $P$ with edges $\ell_{0}$ and $\ell_{1}$.

Now, suppose that there are leaves $\ell^{\prime}$ between $\ell_{0}$ and $\ell_{1}$ with $\sigma_{d}\left(\ell^{\prime}\right)=\ell$. Let $K$ be the collection of all such leaves $\ell^{\prime}$ together with $\ell_{0}$ and $\ell_{1}$. By the above we can form collapsing polygons for each pair of adjacent leaves from $K$. If we unite them and erase in that union all leaves of $K$ except for $\ell_{0}$ and $\ell_{1}$, we will get a collapsing polygon $P$ with edges $\ell_{0}$ and $\ell_{1}$ (leaves of $K$ are diagonals of $P$ ). This proves the main claim of the lemma. Let $G$ be any gap of $\mathcal{L}$ contained in $P$ and with edge $\overline{v x}$. Then $\sigma^{*}(G)=\sigma_{d}(\overline{v x})$ and, hence, $G$ is critical.

We need the following definition.

Definition 3.14. Given a leaf $\ell=\overline{x y}$, we define the corresponding open leaf to be $\ell^{\circ}=\ell \backslash\{x, y\}$. For a lamination $\mathcal{L}$, denote its critical leaves by $\bar{c}_{i}(\mathcal{L})=\bar{c}_{i}$. Below we often consider the set $\bigcup_{i} \bar{c}_{i}^{\circ}$ which is the union of all open critical leaves of $\mathcal{L}$.

Lemma 3.15. Let $\mathcal{L}$ be a sibling d-invariant lamination and $\ell=\overline{a b} \in \mathcal{L}$ be a leaf. If $C$ is a component of $\left\{\left(\sigma_{d}^{*}\right)^{-1}(\ell) \backslash \bigcup_{i} \bar{c}_{i}^{\circ}\right\}$ and $G$ is the convex hull of $\partial(C)$, then $G$ is a leaf or a collapsing polygon of $\mathcal{L}$.

Proof. If $C$ is not a leaf, then there exists $x \in \partial(C)$ which is a vertex of at least two leaves from $C$. Choose leaves $\ell^{\prime}$ and $\ell^{\prime \prime}$ in $C$ which form an angle containing all other leaves from $C$ with the endpoint $x$. By Lemma $3.13 \ell^{\prime}$ and $\ell^{\prime \prime}$ are edges of a collapsing polygon $P$. By properties of laminations all other leaves of $C$ with an endpoint $x$ (if any) are diagonals of $P$. Choose a collapsing polygon $P$, which is maximal by inclusion, with edges $\ell^{\prime}$ and $\ell^{\prime \prime}$.

Let $y$ be a vertex of $P$. Suppose that a leaf $\overline{y z} \subset C$ comes out of $y$ and is not contained in $P$. By properties of a lamination then $\overline{y z}$ is in fact disjoint from $P$ (except for $y$ ). Choose the edge $\ell^{\prime \prime \prime}$ of $P$ with an endpoint $y$ so that the triangle formed by the convex hull of $\ell^{\prime \prime \prime}$ and $\overline{y z}$ is disjoint from the interior of $P$. Then by Lemma 3.13 there exists a collapsing polygon $P^{\prime}$ with edges $\overline{y z}$ and $\ell^{\prime \prime \prime}$. It 

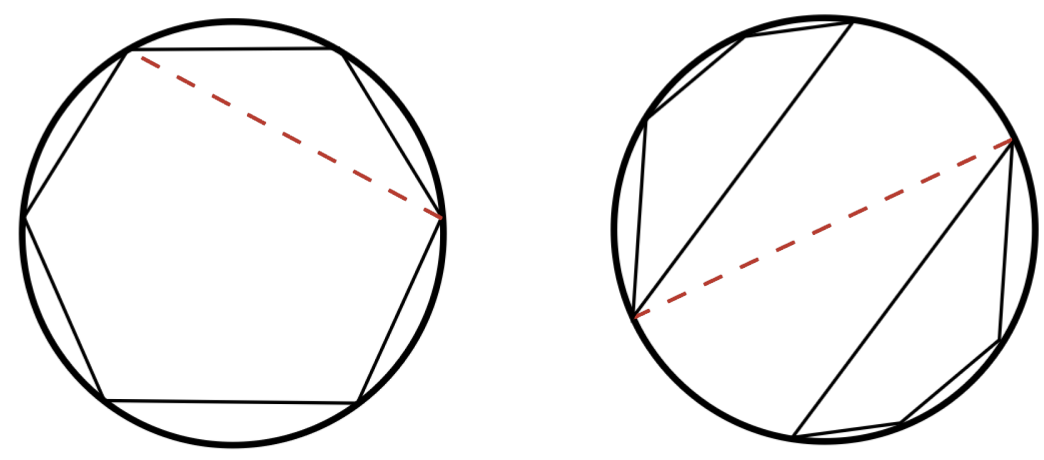

FiguRE 3. Placements of critical leaves

In each picture, the critical leaf is denoted by a dashed line. Notice that in the first example, removing the open critical leaf does not disconnect the polygon,

while in the second example, removing the open critical leaf disconnects a previously connected set, resulting in two components.

follows that $P \cup P^{\prime}$ with $\ell^{\prime \prime \prime}$ removed is a collapsing polygon strictly containing $P$, a contradiction. Thus, $P=G$, as desired.

From now on, by $P_{1}(\ell), \ldots, P_{k}(\ell)$, with $\ell=\overline{a b}$ non-degenerate, we mean the components of $\left\{\left(\sigma_{d}^{*}\right)^{-1}(\ell) \backslash \bigcup_{i} \bar{c}_{i}^{\circ}\right\}$; by Lemma 3.15 they are leaves or collapsing polygons. Note that all edges of the sets $P_{i}(\ell)$ are leaves of $\mathcal{L}$. We view the set $\left(\sigma_{d}^{*}\right)^{-1}(\ell)$ as follows. By Definition 3.1, there is a full preimage collection $L$ of $\ell$. The endpoints of its leaves are either $a$-points or $b$-points (depending on whether they map to $a$ or $b$ ). Often these are all the leaves mapped to $\ell$. Yet, there might exist other leaves which map into $\ell$. Some of these leaves map onto $\ell$; a leaf such as that connects an $a$-point from one leaf of $L$ to a $b$-point from another. Some of these leaves are critical and map to $a$ or $b$; a leaf such as that connects two $a$-points (b-points) belonging to distinct leaves from $L$. Consider the sets $P_{1}(\ell), \ldots, P_{k}(\ell)$. There might exist leaves from $\left(\sigma_{d}^{*}\right)^{-1}(\ell)$ inside $P_{i}(\ell)$, however, we often ignore these leaves (which might or might not be critical). There might also exist other leaves connecting sets $P_{i}(\ell)$ and $P_{j}(\ell), i \neq j$. By Lemma 3.15 such leaves must be critical.

Lemma 3.16. Suppose that $\overline{x v_{0} \cdots v_{k} y}=M$ is an ordered concatenation of leaves of $\mathcal{L}$ such that $\sigma_{d}\left(v_{i}\right)=w$ for all $i$ and $\sigma_{d}(x)=\sigma_{d}(y) \neq w$. Then there exists a collapsing polygon $P_{i}\left(\sigma_{d}^{*}\left(\overline{x v_{0}}\right)\right)$ which contains $M$.

Proof. We see that $\overline{x v_{0}}$ and $\overline{v_{k} y}$ have the same non-degenerate image, while all leaves $\overline{v_{0} v_{1}}, \overline{v_{1} v_{2}}, \ldots$ are critical. Assume that $x=x_{0}<v_{0}<\cdots<v_{k}<y=y_{0}$. Applying Lemma 3.8 to the leaf $\overline{x_{0} v_{0}}$ and its full sibling collection, we obtain a point $x_{1}, v_{0}<x_{1}<v_{1}$ and a leaf $\overline{x_{1} v_{1}}$ which is a sibling of $\overline{x_{0} v_{0}}$. Similarly we obtain points $x_{2}, \ldots, x_{k}$ located between points $v_{1}, v_{2}, \ldots, v_{k}$ and leaves $\overline{x_{i} v_{i}}$ which are siblings of $\overline{x_{0} v_{0}}$.

Now, apply Lemma 3.13 to the leaves $\overline{x_{k} v_{k}}$ and $\overline{v_{k} y_{0}}$. Then there exists a collapsing polygon $P_{0}$ with these leaves as edges. It follows that $v_{k-1}$ is a vertex of $P_{0}$ and there is an ordered concatenation of siblings of $\overline{y_{0} v_{k}}$ which begins with $\overline{y_{0} v_{k}}$ and ends with some leaf $\overline{y_{1} v_{k-1}}$. We can pair this leaf up with the leaf $\overline{x_{k-1} v_{k-1}}$ and 
apply the same arguments. In this manner we will discover a "long" ordered concatenation of siblings which begins with $\overline{y_{0} v_{k}}$ and ends with $\overline{v_{0} x_{0}}$. By Lemma 3.15 there exists a collapsing polygon $P_{i}\left(\sigma_{d}^{*}\left(\overline{x v_{0}}\right)\right)$ from the collection of collapsing polygons described in Lemma 3.15 which contains this concatenation. It follows that $M \subset P_{i}\left(\sigma_{d}^{*}\left(\overline{x v_{0}}\right)\right)$, as desired.

A lamination is called gap-free if it has no gaps. In the next few lemmas we study such laminations. Lemma 3.17 is left to the reader.

Lemma 3.17. Suppose that $\mathcal{L}$ is a gap-free lamination. Then $\mathcal{L}$ does not contain a collection of leaves with one common endpoint such that their other endpoints fill up an arc $I \subset \mathbb{S}$.

A continuous interval map $f: I \rightarrow I$ is called a $d$-sawtooth map if it has $d$ intervals of monotonicity of length $\frac{1}{d}$ and the slope on each such interval is $\pm d$.

Lemma 3.18. If $\mathcal{L}$ is gap-free, then it consists of a family of pairwise disjoint parallel leaves which fill up the entire disk $\overline{\mathbb{D}}$ except for two diametrically opposed points $a, b \in \mathbb{S}$. The factor map $p$ which collapses each leaf to a point, semiconjugates $\sigma_{d}$ to a d-sawtooth map.

Proof. If leaves $\ell_{0}, \ell_{1} \in \mathcal{L}$ have a common endpoint, then by Lemma 3.17 we may assume that $\ell_{0}, \ell_{1}$ form a wedge with no leaves of $\mathcal{L}$ in it and that $\mathcal{L}$ is not gap-free, a contradiction. Hence all leaves are pairwise disjoint. Consider an equivalence relation on $\mathbb{D}$ identifying every leaf into one class. The absence of gaps implies that the quotient space is an interval $I$ and there are only two points $a, b \in \mathbb{S}$ which are not endpoints of leaves. Moreover, if $p: \mathbb{S} \rightarrow I$ is the corresponding factor map, then $p(a), p(b)$ are the endpoints of $I$. Clearly, all leaves of $\mathcal{L}$ must cross the chord $\overline{a b}$ because all points of $I$ separate $p(a)$ and $p(b)$.

Since $\mathcal{L}$ is invariant, then either $\sigma_{d}(a)=a, \sigma_{d}(b)=b$, or $\sigma_{d}(a)=b, \sigma_{d}(b)=a$, or $\sigma_{d}(a)=\sigma_{d}(b)=a$, or $\sigma_{d}(a)=\sigma_{d}(b)=b$. Consider the case when $\sigma_{d}(a)=$ $a, \sigma_{d}(b)=b$ (others are similar). By continuity it follows that as we travel from $a$ to $b$ along the leaves of $\mathcal{L}$ we meet critical leaves $\bar{c}_{1}, \bar{c}_{2}, \ldots, \bar{c}_{d-1}$ (in this order). Suppose that a critical leaf $\bar{c}_{i}$ maps to an endpoint of a leaf $\ell \in \mathcal{L}$. Then some preimage-leaf of $\ell$ will meet $\bar{c}_{i}$ at one of its endpoints, which is impossible as all leaves are pairwise disjoint. Hence all critical leaves of $\mathcal{L}$ map to $a$ or $b$. On the other hand, all leaves whose images are non-disjoint from $a$ or $b$ must be critical and have images coinciding with $a$ or $b$.

It follows from the first and second paragraphs of the proof that the critical leaves $\bar{c}_{1}=\overline{x_{1} y_{1}}, \ldots, \bar{c}_{d-1}=\overline{x_{d-1} y_{d-1}}$ map alternately to $b$ and $a$ and that their endpoints form the full preimages of $a$ and $b$. Assume that the positive circular order is given by $b, x_{d-1}, \ldots, x_{1}, a, y_{1}, \ldots, y_{d-1}$. Then the arc $\left[x_{2}, a\right)$ maps one-to-one onto $\mathbb{S}$ and hence has length $\frac{1}{d}$, while the same applies to the arc $\left(a, y_{2}\right]$. Continuing in the same manner we will see that all critical leaves are in fact perpendicular to the chord $\overline{a b}$ which in fact is a diameter of $\mathbb{D}$. By pulling critical leaves back we complete the proof in the case when $\sigma_{d}(a)=a, \sigma_{d}(b)=b$.

We are ready to prove the main result of this section.

Proof of Theorem 3.2. Let $G$ be a gap of $\mathcal{L}$. If there are two adjacent leaves on the boundary of $G$ which have the same image, then by Lemma 3.13 the gap $G$ maps to a leaf. Moreover, if there are two leaves in $\operatorname{Bd}(G)$ which have the same 
image and are connected with a finite concatenation of critical leaves in $\operatorname{Bd}(G)$, then by Lemma 3.16 again $G$ maps to a leaf. Hence from now on we may assume that the above two cases do not take place on the boundary of $G$. This implies that the $\sigma_{d}^{*}$-image of the boundary of $G$ is not an arc. Indeed, otherwise we can choose an endpoint $x$ of $\sigma_{d}^{*}(\operatorname{Bd}(G))$ and a point $\hat{x} \in \operatorname{Bd}(G)$ with $\sigma_{d}^{*}(\hat{x})=x$ so that $x \in \mathbb{S}, \hat{x} \in \mathbb{S}$, too. It is easy to see that under the assumptions made in the first paragraph this is impossible.

Now, move a point $t$ along $\operatorname{Bd}(G)$ in the positive direction (we can start this movement at an edge $\overline{a b}$ of $G$ ) and consider the movement of its image $\sigma_{d}^{*}$. By Lemma 3.11, then the point $\sigma_{d}^{*}(t)$ moves along $\operatorname{Bd}(G)$ in the positive direction. The first time the point $\sigma_{d}^{*}(t)$ comes back to the already covered part of $\sigma_{d}^{*}(\operatorname{Bd}(G))$ must be at the point $\sigma_{d}^{*}(a)$ because otherwise by Lemma 3.12 there will have to exist leaves of $\mathcal{L}$ inside $G$. Similarly, as the point $t$ keeps moving, its image cannot veer off its previous path because then, again by Lemma 3.12, there will have to exist leaves of $\mathcal{L}$ inside $G$. This proves Theorem 3.2

The basic property defining $d$-invariant sibling laminations is that every noncritical leaf can be extended to a collection of $d$ pairwise disjoint leaves with the same image (a full sibling collection). We conclude this subsection by showing that this implies the same result for arcs as long as their images are monotone arcs.

Lemma 3.19. Suppose that $\sigma_{d}^{*}$ homeomorphically maps an arc $A \sqsubset \mathcal{L}^{*}$ to a monotone arc $B \sqsubset \mathcal{L}^{*}$. Then there are $d$ pairwise disjoint arcs $A_{1}=A, A_{2}, \ldots, A_{d}$ such that for each $i$ the map $\sigma_{d}^{*}$ homeomorphically maps the arc $A_{i}$ to $B$.

Proof. Suppose that the endpoints of $B$ are $p, q$ and that $B \subset[p, q]=I$. Denote by $J_{1}, \ldots, J_{d}$ circle arcs which map one-to-one to $I$. Since $B$ is an arc, there are no more than countably many leaves in $B$. We can order them so that they form a sequence of leaves $\ell_{n} \subset B$ with $\operatorname{diam}\left(\ell_{n}\right) \rightarrow 0$. Given a leaf $\ell_{n} \subset B$ we can choose its unique preimage $\ell_{n}^{1} \subset A$ and then choose a full sibling collection of $\ell_{n}^{1}$ consisting of leaves $\ell_{n}^{1}, \ell_{n}^{2}, \ldots, \ell_{n}^{d}$. In other words, we choose a full preimage collection of leaves for each leaf from $B$ so that this preimage collection includes a leaf from $A$. Call $\ell_{n}$ long if there exists a leaf $\ell_{n}^{j}$ with endpoints coming from distinct sets $J_{r}$ and $J_{s}$.

We claim that there are no more than finitely many long leaves in $B$. Indeed, suppose otherwise. By continuity we can then choose a subsequence for which we may assume that 1 ) there is a sequence of leaves $t_{n} \subset B$ which converges to a point $x \in \mathbb{S}$ from one side, and 2) there is a sequence of their pullback-leaves $\hat{t}_{n}$ (i.e., $\left.\sigma_{d}^{*}\left(\hat{t}_{n}\right)=t_{n}\right)$ which converges to a critical leaf $\hat{x}$ from one side. Clearly, this is impossible since $B$ is a monotone arc.

Suppose that $\ell_{n_{1}}=\overline{a_{n_{1}} b_{n_{1}}}, \ldots, \ell_{n_{k}}=\overline{a_{n_{k}} b_{n_{k}}}$ are all long leaves in $B$. Without loss of generality we may assume that $a_{n_{1}}<b_{n_{1}} \leq a_{n_{2}}<\cdots<b_{n_{k}}$. Denote closures of components of $B \backslash \bigcup \ell_{n_{i}}$ by $S_{1}, S_{2}, \ldots, S_{k+1}$ numbered in the natural order on the circle so that $S_{1}$ precedes $a_{n_{1}}, S_{2}$ is located between $b_{n_{1}}$ and $a_{n_{2}}$, etc. (some of these sets may be empty, e.g. if $p=a_{n_{1}}$, then $S_{1}$ is empty).

Then each $S_{j}$ has $d$ pullbacks, each of which is a monotone $\operatorname{arc} \widehat{S}_{j}^{r}$ such that $\partial\left(\widehat{S}_{j}^{r}\right) \subset I_{r}$. Let us consider the union of all such pullbacks with all leaves from previously chosen full preimage collections of all leaves $\ell_{n_{1}}, \ldots, \ell_{n_{k}}$ (i.e., of all long leaves in $B$ ). Basically, we consider all preimage collections of leaves of $B$ and then take the closure of their union, representing it in a convenient form. We claim 
that each component of this union is a monotone arc which maps onto $B$ in a oneto-one fashion. Indeed, by construction each such component $X$ can be extended both clockwise and counterclockwise until it reaches points mapped to $p$ and $q$ respectively. It follows from the construction that these components are pairwise disjoint and that one of them is the originally given arc $A=A_{1}$. This completes the proof.

3.3. The space of all sibling invariant laminations. Our approach is to describe laminations in the "language of leaves". The main idea is to use sibling invariant laminations for that purpose. By Theorem 3.2 this does not push us outside the class of Thurston invariant laminations. According to the philosophy explained in the Introduction, now we need to verify if the set of all sibling invariant laminations contains all q-laminations and is Hausdorff closed. The first step here is made in Lemma 3.20 in which we relate sibling invariant laminations and q-laminations. Observe that it is well known (and easy to prove) that $d$-invariant q-laminations are Thurston $d$-invariant laminations.

Lemma 3.20. $d$-invariant q-laminations are sibling d-invariant.

Proof. Assume that $\sim$ is a $d$-invariant laminational equivalence relation. Conditions (1) and (2) of Definition 3.1 are immediate. To check condition (3) of Definition 3.1. assume that $\ell$ is a non-critical leaf of $\mathcal{L}_{\sim}$ and verify that there are $d$ pairwise disjoint leaves $\ell=\ell_{1}, \ldots, \ell_{d}$ with the same image. To do so, consider the collection $\mathcal{A}$ of all $\sim$-classes which map to the $\sim$-class of $\sigma_{d}(\ell)$. If a $\sim$-class $\mathfrak{g} \in \mathcal{A}$ does not contain $\ell$ and is not critical, then $\operatorname{Bd}(\mathfrak{g})$ contains only a unique sibling of $\ell$. If $\mathfrak{g}$ is critical, it maps to the $\sim$-class of $\sigma_{d}(\ell)$, say, $k$-to- 1 , and we can choose $k$ pairwise disjoint sibling leaves of $\ell$ on the boundary of $\mathrm{CH}(\mathfrak{g})$. If $\ell$ is an edge of the set $\mathrm{CH}(\mathfrak{h})$ where $\mathfrak{h}$ is a critical class, we can still find the appropriate number of its pairwise disjoint siblings in the boundary of $\mathrm{CH}(\mathfrak{h})$. The endpoints of leaves from the thus-created list exhaust the list of all points which are preimages of endpoints of $\sigma_{d}(\ell)$. Thus, we get $d$ siblings, one of which is $\ell$, as desired.

We will now prove that all limits of q-laminations are sibling $d$-invariant. Thus, if there is a Thurston $d$-invariant lamination $\mathcal{L}$ which is not sibling $d$-invariant, then it is not a Hausdorff limit of q-laminations. This shows that the class of Thurston $d$-invariant laminations is too wide if we are interested in Hausdorff limits of qlaminations. The above justifies our interest in the next example illustrated in Figure 4

Suppose that points $\hat{x}_{1}, \hat{y}_{1}, \hat{z}_{1}, \hat{x}_{2}, \hat{y}_{2}, \hat{z}_{2}$ are positively ordered on $\mathbb{S}$ and that $H=\mathrm{CH}\left(\hat{x}_{1} \hat{y}_{1} \hat{z}_{1} \hat{x}_{2} \hat{y}_{2} \hat{z}_{2}\right)$ is a critical hexagon of an invariant q-lamination $\mathcal{L}$ such that $\sigma_{d}^{*}: H \rightarrow T$ maps $H$ in the 2-to- 1 fashion onto the triangle $T=\mathrm{CH}(x y z)$ with $\sigma_{d}\left(\hat{x}_{i}\right)=x, \sigma_{d}\left(\hat{y}_{i}\right)=y, \sigma_{d}\left(\hat{z}_{i}\right)=z$. Now, add to the lamination $\mathcal{L}$ the leaves $\overline{\hat{x}_{1} \hat{z}_{1}}$ and $\overline{\hat{x}_{1} \hat{x}_{2}}$ and all their pullbacks along the backward orbit of $H$ under $\sigma_{d}^{*}$. Denote the thus-created lamination $\mathcal{L}^{\prime}$. It is easy to see that $\mathcal{L}^{\prime}$ is Thurston $d$-invariant but not sibling $d$-invariant because $\overline{\hat{x}_{1} \hat{z}_{1}}=\ell$ cannot be completed to a full sibling collection (clearly, $H$ does not contain siblings of $\ell$ ).

Lemma 3.21. Take sequences of d sibling leaves $\overline{\hat{a}_{i}^{j} \hat{b}_{i}^{j}}, 1 \leq j \leq d, i=1,2, \ldots$, such that $\overline{\hat{a}_{i}^{1} \hat{b}_{i}^{1}} \rightarrow \overline{\hat{a}^{1} \hat{b}^{1}}=\ell^{1}, \overline{\hat{a}_{i}^{2} \hat{b}_{i}^{2}} \rightarrow \overline{\hat{a}^{2} \hat{b}^{2}}=\ell^{2}, \ldots, \overline{\hat{a}_{i}^{d} \hat{b}_{i}^{d}} \rightarrow \overline{\hat{a}^{d} \hat{b}^{d}}=\ell^{d}$ and $\sigma_{d}\left(\ell^{1}\right)$ is not degenerate. Then $\ell^{j}, 1 \leq j \leq d$ are siblings with non-degenerate image. 


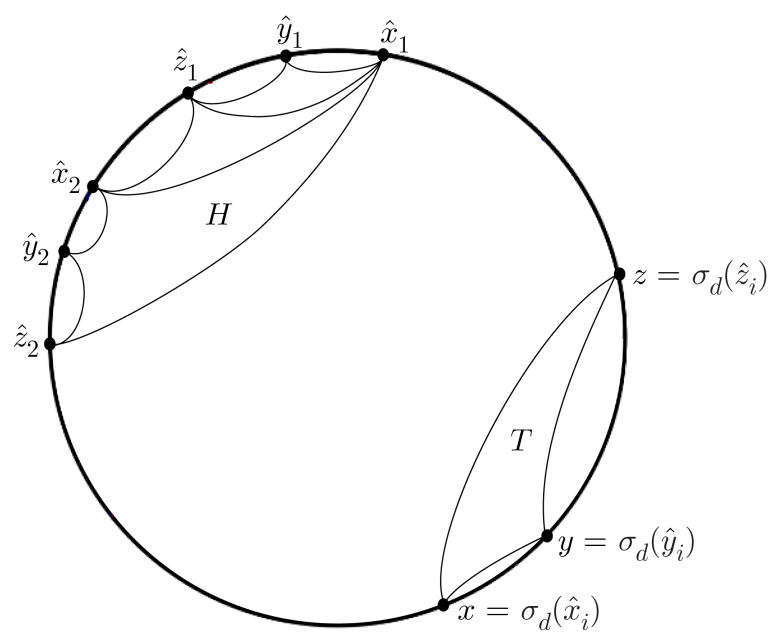

Figure 4. An example of a Thurston invariant lamination which is not sibling invariant. The leaf $\overline{\hat{x}_{1} \hat{z}_{1}}$ has no siblings in $H$.

Proof. By continuity, $\sigma_{d}\left(\ell^{j}\right)=\sigma_{d}\left(\ell^{1}\right)$ for all $j$. To show that leaves $\ell^{j}, 1 \leq j \leq d$ are pairwise disjoint consider $i_{0}$ and $\varepsilon>0$ such that for each $i \geq i_{0}$ and each $j$, $1 \leq j \leq d$ we have that $\left|\overline{\hat{a}_{i}^{j} \hat{b}_{i}^{j}}\right| \geq \varepsilon$. Then it follows that for a fixed $i$ the pairwise distance between points of sets $\left\{\hat{a}_{i}^{j}, \hat{b}_{i}^{j}\right\}$, for $1 \leq j \neq k \leq d$, is bounded away from 0 $\left(\hat{a}_{i}^{j}, \hat{a}_{i}^{k}\right.$ cannot be too close because their images coincide and $\hat{a}_{i}^{j}, \hat{b}_{i}^{k}$ cannot be too close because their images are too far apart). Hence the leaves $\ell^{j}, 1 \leq j \leq d$ are disjoint, as desired.

Suppose that $\mathcal{L}$ is a prelamination. Then by its closure $\overline{\mathcal{L}}$ we mean the set of chords in $\overline{\mathbb{D}}$ which are limits of sequences of leaves of $\mathcal{L}$. It is easy to see that $\overline{\mathcal{L}}$ is a closed lamination. Corollary 3.22 shows that it is enough to verify the property of being sibling invariant on dense prelaminations. It immediately follows from Lemma 3.21 (so it is given here without proof).

Corollary 3.22. If $\mathcal{L}$ is a sibling d-invariant prelamination, then its closure $\overline{\mathcal{L}}$ is a sibling d-invariant lamination.

Theorem 3.23 follows from Lemma 3.20 and Lemma 3.21 .

Theorem 3.23. The Hausdorff limit of a sequence of sibling invariant laminations is a sibling invariant lamination. The space of all sibling invariant laminations is closed in the Hausdorff sense and contains all q-laminations.

\section{Proper laminations}

Clearly, not all (sibling, Thurston) invariant laminations are q-laminations (e.g., a lamination with two finite gaps with a common edge is not a q-lamination). In this section we address this issue and describe Thurston $d$-invariant laminations which almost coincide with appropriate q-laminations. According to the adopted approach, we use the "language of leaves" in our description.

Definition 4.1 (Proper lamination). Two leaves with a common endpoint $v$ and the same image which is a leaf (and not a point) are said to form a critical wedge 
(the point $v$ is then said to be its vertex). A lamination $\mathcal{L}$ is proper if it contains no critical leaf with periodic endpoint and no critical wedge with periodic vertex.

Proper laminations are instrumental for our description of laminations which almost coincide with q-laminations.

Lemma 4.2. Any q-lamination is proper.

Proof. Suppose that $A$ is either a critical wedge or a critical leaf which contains a periodic point of period $n$. Then $A$ is contained in a finite class $\mathfrak{g}$ such that $\left|\sigma_{d}(\mathfrak{g})\right|<$ $|\mathfrak{g}|$, while on the other hand $\sigma_{d}^{n}(\mathfrak{g})$ must coincide with $\mathfrak{g}$, a contradiction.

The exact inverse of Lemma 4.2 fails. However, it turns out that proper laminations are very close to q-laminations. To show this we need a few technical definitions.

Definition 4.3. Suppose that $A$ is a polygon with vertices in $\mathbb{S}$. It is said to be $d$-wandering if for any $m \neq n$ we have $\mathrm{CH}\left(\sigma_{d}^{m}(A \cap \mathbb{S})\right) \cap \mathrm{CH}\left(\sigma_{d}^{n}(A \cap \mathbb{S})\right)=\emptyset$.

In Definition 4.3 we do not require that $A$ be a part of some lamination or even that the circular orientation of vertices of $A$ be preserved under $\sigma_{d}$. Still, it follows from Kiwi's results Kiw02 (see also a later paper by Childers [Chi07]) that $A$ cannot have too many vertices.

Theorem 4.4 ([Kiw02, Chi07]). Suppose that $A$ is a polygon with more than $d^{d}$ vertices. Then it is not d-wandering.

In Definition 4.3 we assume that images of $A$ have pairwise disjoint convex hulls. As Lemma 4.5 shows, one can slightly weaken this condition and still obtain useful conclusions.

Lemma 4.5. Suppose that one of the following holds.

(1) $A$ is a polygon, $\partial(A) \subset \mathbb{S}$, and for any $m \neq n$ the interiors of the convex hulls $\mathrm{CH}\left(\sigma_{d}^{m}(A \cap \mathbb{S})\right)$ and $\mathrm{CH}\left(\sigma_{d}^{n}(A \cap \mathbb{S})\right)$ are disjoint;

(2) $A$ is a chord of $\mathbb{S}$, and for any $m \neq n$ two image chords $\left(\sigma_{d}^{*}\right)^{m}(A)$ and $\left(\sigma_{d}^{*}\right)^{n}(A)$ are disjoint inside $\mathbb{D}$.

Then, if $\left.\sigma_{d}^{n}\right|_{\partial(A)}$ is one-to-one for all $n$, then either $A$ is wandering or $A$ is a chord with a preperiodic endpoint.

Proof. Suppose that $A=\overline{p q}$ is a non-wandering chord and $p, q$ have infinite orbits. We may assume that $\sigma_{d}^{*}(A)=\overline{q r}$ and $\sigma_{d}(p)=q$. Set $A_{k}=\left(\sigma_{d}^{*}\right)^{k}(A)$. Take closures of the two components of $\mathbb{D} \backslash A_{k}$. By the assumptions, $\bigcup_{i>k} A_{i}$ is contained in one of them. Then it is easy to see that $A_{i}$ converges to either a $\sigma_{d}$-fixed point on $\mathbb{S}$ or a $\sigma_{d}^{*}$-invariant chord. However, this contradicts the fact that $\sigma_{d}$ is repelling.

Now suppose that $A$ is a non-wandering polygon. We may assume that $\sigma_{d}^{*}(A)$ and $A$ intersect either (1) at a common vertex $x$ or (2) along a common edge $\ell=\overline{x y}$. Choose the vertex $u$ of $A$ with $\sigma_{d}(u)=x$ and consider the chord $\overline{u x}$. The chord $\overline{u x}$ satisfies the assumptions of the theorem and is non-wandering. Then by the above $u$ is preperiodic. We may assume that $u$ is fixed. Consider the two edges $\overline{u v_{0}}$ and $\overline{u w_{0}}$ of $A$ and the arc $\left[v_{0}, w_{0}\right]$ in $\mathbb{S}$ not containing $u$. Similarly, for each $n$ set $v_{n}=\sigma_{d}^{n}\left(v_{0}\right)$ and $w_{n}=\sigma_{d}^{n}\left(w_{0}\right)$. Since the interiors of the convex hulls of $\sigma_{d}^{n}(A)$ are pairwise disjoint, the open $\operatorname{arcs}\left(v_{n}, w_{n}\right)$ are also pairwise disjoint, and hence their diameter must converge to zero, a contradiction to the fact that $\sigma_{d}$ is expanding. 
Lemma 4.6. Suppose that $\mathcal{L}$ is a Thurston invariant lamination. Then there are at most finitely many points $x$ such that there is a critical leaf with an endpoint $x$ or a critical wedge with vertex $x$.

Proof. Clearly there are at most finitely many critical leaves. Hence we may suppose that there are infinitely many points $x_{i}$ such that there are leaves $\overline{a_{i} x_{i}}, \overline{b_{i} x_{i}}$ with $\sigma_{d}\left(a_{i}\right)=\sigma_{d}\left(b_{i}\right) \neq \sigma_{d}\left(x_{i}\right)$. We may assume that the sets $\overline{\sigma_{d}\left(a_{i}\right) \sigma_{d}\left(x_{i}\right)}$ are all distinct and the points $\sigma_{d}\left(x_{i}\right)$ are all distinct. Clearly, the chords $\overline{a_{i} b_{i}}$ and $\overline{a_{j} b_{j}}$ are disjoint inside $\mathbb{D}$. Since each such chord is critical, we have a contradiction.

Let $E(v)$ be the set of all endpoints of leaves with a common endpoint $v$ (if $E(v)$ accumulates upon $v$ we include $v$ in $E(v)$ ). Then $E(v)$ is a closed set. Let $C(v)$ be the family of all leaves connecting $v$ and a point of $E(v)$ (it might include $\{v\}$ as a degenerate leaf).

Lemma 4.7. Suppose that $v$ is a point with infinite orbit and $\mathcal{L}$ is a Thurston $d$ invariant lamination. Then there are at most finitely many leaves with an endpoint $v$.

Proof. Let $E(v)$ be infinite. By Lemma4.6, we may assume that $v$ and all its images are not endpoints of critical leaves or vertices of critical wedges. If $v$ ever maps to $E(v)$, then by Lemma $4.5 v$ is preperiodic, a contradiction. Choose $A \subset E(v)$ consisting of $d^{d}$ points. Consider $\mathrm{CH}(A \cup\{v\})$. By the above for any $n \neq m$, the interiors of $\mathrm{CH}\left(\sigma_{d}^{n}(A \cup\{v\})\right)$ and $\mathrm{CH}\left(\sigma_{d}^{m}(A \cup\{v\})\right)$ are disjoint. Then by Lemma4.5 $\mathrm{CH}(A \cup\{v\})$ is wandering, contradicting Lemma 4.4

Lemma 4.8. Suppose that $\mathcal{L}$ is a Thurston d-invariant lamination and $A=$ $\overline{x_{0} \cdots x_{i} x_{i+1} \cdots}, i=0,1, \ldots$, is a concatenation of leaves of $\mathcal{L}$ (the set $\partial(A)$ is infinite). Then $A$ has preperiodic vertices.

Proof. Consider the convex hulls of sets $\overline{\partial(A)}=B_{0}, \overline{\partial\left(\sigma_{d}(A)\right)}=B_{1}, \ldots$ Suppose that all such convex hulls have disjoint interiors. There may exist numbers $n$ such that $\left.\sigma_{d}\right|_{B_{n}}$ is not one-to-one. This means that for each such $n$ there is a critical chord $\ell_{n}$ inside the convex hull of $B_{n}$. Since we assume that the interiors of sets $\mathrm{CH}\left(B_{n}\right)$ are disjoint, one critical chord can correspond to at most two sets $B_{n}$; otherwise two critical chords $\ell_{n}$ and $\ell_{m}$ cannot intersect. It follows that there are at most finitely many critical chords $\ell_{i}$ constructed as above and that for large enough $n$ the map $\left.\sigma_{d}\right|_{B_{i}}$ is one-to-one. By Lemma 4.5 this is impossible. Hence convex hulls of sets $B_{n}$ have non-disjoint interiors. Since leaves of $\mathcal{L}$ cannot intersect inside $\mathbb{D}$, this implies that we can make the following assumption: there exists $m, n$ such that $\sigma_{d}^{m}\left(x_{0}\right)=x_{n}$. We may also assume that $x_{n} \neq x_{0}$.

Set $C=\overline{x_{0} x_{1} \cdots x_{n-1} x_{n}}$. Then $\sigma_{d}(C)$ is a concatenation of leaves attached to $C$, etc. If for some $k$ the set $\sigma_{d}^{k}(C)$ is a point we can choose the minimal such $k$ which implies that $\sigma_{d}^{k-1}(C)$ is a concatenation of leaves whose image is one of its own vertices $y$. Hence $y$ is periodic as desired. Otherwise we may assume that the number of vertices of $C$ does not drop under iterations of $\sigma_{d}$.

If $C$ has self-intersections, we may refine $C$ to get a concatenation with no self-intersections still connecting $x_{0}$ and $x_{n}$. We can optimize the situation even more. Indeed, choose the shortest (in terms of the number of its leaves) subchain $D$ of $C$ which ever intersects itself. Then it follows that the image $\sigma_{d}^{k}(D)$ of $D$ which intersects $D$ must be concatenated to $D$ at its endpoint (otherwise $D$ can 
be shortened even further). Thus, if there are no preperiodic vertices of $D$, then we get a sequence of $\sigma_{d}^{k}$-images of $D$ concatenated to each other at their ends. It now follows that $\lim \sigma_{d}^{n}(D)$ is either a leaf in $\mathcal{L}$ or a point of $\mathbb{S}$. In either case this contradicts that $\sigma_{d}$ is expanding.

Recall that $\approx_{\mathcal{L}}$ was the equivalence relation defined by $x \approx_{\mathcal{L}} y$ if and only if there exists a finite concatenation of leaves of $\mathcal{L}$ connecting $x$ and $y$. Theorem 4.9 specifies properties of $\approx_{\mathcal{L}}$.

Theorem 4.9. Let $\mathcal{L}$ be a proper Thurston invariant lamination. Then $\approx_{\mathcal{L}}$ is an invariant laminational equivalence relation.

Proof. Let us show that any point $v \in \mathbb{S}$ is an endpoint of at most finitely many leaves of $\mathcal{L}$. Otherwise by Lemma 4.7 we may assume that $v$ is fixed. Take the infinite invariant set $E^{\prime}=E(v) \cup\{v\}$. Since $\sigma_{d}$ is expanding and because there are no infinite compact spaces admitting one-to-one expanding maps, $E^{\prime}$ contains points $x, x^{\prime}$ with $\sigma_{d}(x)=\sigma_{d}\left(x^{\prime}\right)$ contradicting the fact that $\mathcal{L}$ is proper.

Suppose next that $A=\overline{x_{0} \cdots x_{i} x_{i+1} \cdots}, i=0,1, \ldots$, is an infinite concatenation of leaves of $\mathcal{L}$. By Lemma 4.8 we may assume that $x_{0}=x$ is fixed. Let us show that if $\ell_{1}=\overline{x e_{1}}, \ldots, \ell_{k}=\overline{x e_{k}}$ are all the leaves with the endpoint $x$, then $\sigma_{d}\left(e_{i}\right)=e_{i}$ for all $i$. Since $\mathcal{L}$ is proper, the leaves $\ell_{1}, \ldots, \ell_{k}$ have distinct non-degenerate $\sigma_{d}^{*}$ images. Hence all points $e_{i}, 1 \leq i \leq k$ are periodic. If $k=1$, then $e_{1}$ is fixed and we are done. If $k=2$, then $\ell_{1}, \ell_{2}$ are edges of some gap $G$, and the fact that the orientation is preserved under $\sigma_{d}$ implies that both $\ell_{1}, \ell_{2}$ are fixed. Suppose that $k \geq 3$. We may assume that $e_{1}<e_{2}<\cdots<e_{k}$. Since gaps map to gaps and the orientation is preserved on them, the fact that $x$ is fixed implies that then $\sigma_{d}\left(e_{1}\right)<\sigma_{d}\left(e_{2}\right)<\cdots<\sigma_{d}\left(e_{k}\right)$ and hence in fact $\sigma_{d}\left(e_{i}\right)=e_{i}, 1 \leq i \leq k$. Thus, the leaf $\overline{x_{0} x_{1}}$ is fixed, the leaf $\overline{x_{1} x_{2}}$ is fixed and, by induction, all the leaves $\overline{x_{i} x_{i+1}}$ are fixed, a contradiction.

By the above $\mathcal{L}$ contains no infinite cones and no infinite concatenations of leaves. Let us show that all $\approx_{\mathcal{L}^{-}}$classes are finite (and hence closed). Otherwise let $E$ be an infinite $\approx_{\mathcal{L}}$-class and let $x_{0} \in E$. For each $y \in E$ fix a concatenation of leaves $L_{y}$ from $x_{0}$ to $y$ containing the least number of leaves. Then there are infinitely many sets $L_{y}, y \in E$. Since there are only finitely many leaves of $\mathcal{L}$ with an endpoint $x_{0}$, we can choose $x_{1} \in E$ so that there are infinitely many sets $L_{y}, y \in E$ whose first leaf is $\overline{x_{0} x_{1}}$. Since there only finitely many leaves of $\mathcal{L}$ with an endpoint $x_{1}$, we can choose $x_{2} \in E$ so that there are infinitely may sets $L_{y}, y \in E$ whose second leaf is $\overline{x_{1} x_{2}}$. Continuing in this manner we will find an infinite concatenation of leaves of $\mathcal{L}$, a contradiction (by the choice of sets $L_{y}, y \in E$, the points $x_{0}, x_{1}, \ldots$ are all distinct).

Take convex hulls of $\approx_{\mathcal{L}}$-classes. Clearly, these convex hulls are pairwise disjoint. It follows that if a non-constant sequence of $\approx_{\mathcal{L}}$-classes converges, then it converges to a leaf of $\mathcal{L}$ or a point. Hence $\approx_{\mathcal{L}}$ is a closed equivalence relation. To show that $\approx_{\mathcal{L}}$ is invariant and laminational we have to prove that $\approx_{\mathcal{L}}$-classes map onto $\approx_{\mathcal{L}^{-}}$-classes in a covering way (i.e., we need to check conditions (D1) and (D3) of Definition 2.2). Let us show that for any $x \in \mathbb{S}$ the $\approx_{\mathcal{L}^{-}}$class maps onto the $\approx_{\mathcal{L}^{-}}$ class of $\sigma_{d}(x)$. Indeed, let $y$ belong to the $\approx_{\mathcal{L}^{-}}$-class of $\sigma_{d}(x)$. Choose a finite concatenation $\ell_{1} \ell_{2} \cdots \ell_{k}$ of leaves connecting $\sigma_{d}(x)$ and $y$ (here $x$ is an endpoint of $\ell_{1}$ and $y$ is an endpoint of $\ell_{k}$ ). Take a pullback-leaf $\overline{x x_{1}}$ of $\ell_{1}$ with an endpoint $x$, then a pullback-leaf $\overline{x_{1} x_{2}}$ of $\ell_{2}$, etc., until we get a finite concatenation of leaves 
connecting $x$ and some point $y^{\prime}$ with $\sigma_{d}\left(y^{\prime}\right)=y$. This implies that the $\approx_{\mathcal{L}^{-}}$class

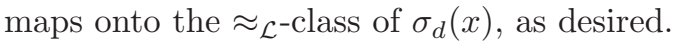

It remains to prove that $\approx_{\mathcal{L}}$ satisfies condition (D3) from Definition 2.2 (i.e., that $\sigma$ is covering on $\approx_{\mathcal{L}}$-classes). Observe that if $\mathcal{L}$ is sibling invariant, this immediately follows from Corollary 3.7. In the case when $\mathcal{L}$ is Thurston invariant we need an extra argument.

So, suppose that $\mathfrak{g}$ is a $\approx_{\mathcal{L}}$-class. We may assume that $|\mathfrak{g}|>2$. Let $\ell$ be an edge of $\mathrm{CH}(\mathfrak{g})$. Then either $(1) \ell$ is approached from the outside of $\mathrm{CH}(\mathfrak{g})$ by leaves $\ell_{i}$ of $\mathcal{L}$ or $(2)$ there is an infinite gap $G$ on the other side of $\ell$, opposite to the side where $\mathrm{CH}(\mathfrak{g})$ is located. Below we refer to these as cases (1) and (2).

Let us show that if one of the edges of $\mathrm{CH}(\mathfrak{g})$ is critical, then all are critical. Indeed, let $\ell=\overline{a b}$ be a critical edge of $\mathrm{CH}(\mathfrak{g})$. In case (1) images of $\ell_{i}$ separate $\sigma_{d}(\ell)$ from the rest of the circle; hence all points of $\mathfrak{g}$ map to the same point, as desired. In case (2) both endpoints of $\ell$ are limit points of vertices of $G$ because otherwise we could extend the $\approx_{\mathcal{L}}$-class $\mathfrak{g}$. Since $\mathcal{L}$ is Thurston invariant, we conclude that $\sigma_{d}(\ell)$ is a vertex of an infinite gap $\sigma_{d}(G)$, and $\sigma_{d}(\ell)$ is approached from either side on $\mathbb{S}$ by vertices of $\sigma_{d}(G)$. Hence no leaves can come out of $\sigma_{d}(\ell)$, and again all points of $\mathfrak{g}$ map to the same point. Thus, in this case Definition 2.2(D3) is satisfied for $\mathrm{CH}(\mathfrak{g})$.

Now suppose that all edges of $\mathrm{CH}(\mathfrak{g})$ are non-critical. We claim that if $\ell$ is an edge of $\mathrm{CH}(\mathfrak{g})$, then $\sigma_{d}(\ell)$ is an edge of $\mathrm{CH}\left(\sigma_{d}(\mathfrak{g})\right)$. Indeed, in case $(1) \sigma_{d}(\ell)$ is approached by leaves of $\mathcal{L}$ from one side, and in case $(2) \sigma_{d}(\ell)$ borders an infinite gap of $\mathcal{L}$ from one side. In either case $\sigma_{d}(\ell)$ cannot be a diagonal of the gap $\mathrm{CH}\left(\sigma_{d}(\mathfrak{g})\right)$, and the claim is proved.

It remains to show that as we walk along the boundary of $\mathrm{CH}(\mathfrak{g})$, the $\sigma_{d}$-image of the point walks in the positive direction along the boundary of $\mathrm{CH}\left(\sigma_{d}(\mathfrak{g})\right)$. Indeed, suppose first that $\sigma_{d}(\mathfrak{g})$ consists of two points. Then by the above there are no critical edges of $\mathrm{CH}(\mathfrak{g})$, and the condition we want to check is automatically satisfied. Otherwise let $\mathrm{CH}\left(\sigma_{d}(\mathfrak{g})\right)$ be a gap. Let $\overline{a b}$ be an edge of $\left.\mathrm{CH}(\mathfrak{g})\right)$ such that moving from $a$ to $b$ along $\overline{a b}$ takes place in the positive direction on the boundary of $\mathrm{CH}(\mathfrak{g})$. Then in case (1) the set $\sigma_{d}(\mathfrak{g})$ must be located so that movement from $\sigma_{d}(a)$ to $\sigma_{d}(b)$ takes place in the positive direction on the boundary of $\mathrm{CH}\left(\sigma_{d}(\mathfrak{g})\right)$ as on the other side of $\sigma_{d}^{*}(\overline{a b})$ images of leaves, approximating $\overline{a b}$, approximate $\sigma_{d}^{*}(\overline{a b})$. In case (2) we make the same conclusion because on the other side of $\sigma_{d}^{*}(\overline{a b})$ the image of an infinite gap adjacent to $\mathrm{CH}(\mathfrak{g})$ along $\overline{a b}$ is located (and this image cannot contain $\mathrm{CH}\left(\sigma_{d}(\mathfrak{g})\right)$. Hence the map is positively oriented on $\mathrm{Bd}(\mathrm{CH}(\mathfrak{g}))$, as desired.

Theorem 4.9 shows that, up to a "finite" restructuring, a lamination is a qlamination if and only it is proper. The appropriate claim is made in Corollary 4.10, whose proof is left to the reader.

Corollary 4.10. A proper Thurston invariant lamination $\mathcal{L}$ is a q-lamination if and only if for each $\approx_{\mathcal{L}}$-class $\mathfrak{g}$ the edges of its convex hull $\mathrm{CH}(\mathfrak{g})$ belong to $\mathcal{L}$, while no leaf of $\mathcal{L}$ is contained in the interior of $\mathrm{CH}(\mathfrak{g})$.

\section{Clean laminations}

Thurston defined clean laminations. In this section we show that every clean Thurston invariant lamination is a proper sibling invariant lamination. Thus, up to 
a minor modification every clean Thurston invariant lamination is a q-lamination. We show in the next section that every clean Thurston 2-invariant lamination is a q-lamination.

Definition 5.1. Let $\mathcal{L}$ be a lamination. Then $\mathcal{L}$ is clean if no point of $\mathbb{S}$ is the common endpoint of three distinct leaves of $\mathcal{L}$.

Theorem 5.2. Let $\mathcal{L}$ be a Thurston d-invariant clean lamination. Then $\mathcal{L}$ is a proper sibling d-invariant lamination.

Proof. Let $\mathcal{L}$ be a clean Thurston $d$-invariant lamination. Suppose first that $\mathcal{L}$ contains a critical leaf $\overline{x y}$ with a periodic endpoint. Assume that $x$ is fixed. There must exist $d$ disjoint leaves which map to $\overline{x y}$. One of them must have $x$ as an endpoint. Label this leaf $\overline{x z}$ (since $\sigma_{d}^{*}(\overline{x y})=x, y \neq z$ ). Similarly, there must exist $d$ leaves which map to $\overline{x z}$; one of them must be $\overline{x w}$ (and, as above, $\overline{x y}, \overline{x z}, \overline{x w}$ are distinct). Hence $\mathcal{L}$ is not clean, a contradiction. The case when $\mathcal{L}$ contains a critical wedge is similar. Thus, $\mathcal{L}$ is proper.

Suppose next that $\ell=\overline{x y} \in \mathcal{L}$ and $\sigma_{d}(\ell)$ is non-degenerate. To show that $\mathcal{L}$ is sibling $d$-invariant we need to show that there are $d-1$ siblings of $\ell$. Since $\mathcal{L}$ is a Thurston $d$-invariant lamination, there exists a collection $B$ of $d$ pairwise disjoint leaves $\ell_{1}, \ldots, \ell_{d}$ so that $\sigma_{d}\left(\ell_{i}\right)=\sigma_{d}(\ell)$ for all $i$. If $\ell=\ell_{i}$ for some $i$, we are done. Otherwise there exists $i \neq j$ so that $\ell_{i} \cap \ell \neq \emptyset \neq \ell_{j} \cap \ell$. Let $\ell_{i}=\overline{x z}, \ell_{j}=\overline{y t}$ and consider two cases.

(1) Points $z$ and $t$ are located in distinct components of $\mathbb{S} \backslash\{x, y\}$. Then $\ell_{i}$ and $\ell$ are edges of a certain gap $G$ because $\mathcal{L}$ is clean. Since $\left.\sigma_{d}^{*}\right|_{\operatorname{Bd}(G)}$ is positively oriented in case $\mathrm{CH}\left(\sigma_{d}(\partial G)\right)$ is a gap, $G$ must be a finite gap of $\mathcal{L}$, collapsing to a leaf. Hence there exists an edge of $G$ with an endpoint $y$, contradicting the assumption that $\mathcal{L}$ is clean.

(2) Points $z$ and $t$ belong to the same component of $\mathbb{S} \backslash\{x, y\}$. Similar to (1), there exists a gap $G$ with edges $\ell_{i}, \ell, \ell_{j}$ (and possibly other edges), collapsed onto $\sigma_{d}(\ell)$ under $\sigma_{d}$. Since $\mathcal{L}$ is clean, every leaf of $\mathcal{L}$, which intersects $G$, is contained in $\operatorname{Bd}(G)$. Hence $\operatorname{Bd}(G)$ consists of $2 n$ leaves all of which map to $\sigma_{d}(\ell)$, and, possibly, to some critical leaves.

Let us show that $G$ has no critical edges. Suppose that $\overline{u v}$ is a critical edge of $G$ such that all vertices of $G$ are contained in the circle arc $I=[v, u]$. Each leaf of $\mathcal{L}$ close to $\overline{u v}$ and with endpoint from $(u, v)$ will have the image which crosses $\sigma_{d}(\ell)$. Hence there are no such leaves and $\overline{u v}$ is an edge of a gap $H$ whose vertices belong to $[u, v]$. Since $\mathcal{L}$ is clean, there are no edges of $H$ with endpoint $u$ or $v$ except for $\overline{u v}$. Hence there exist sequences $u_{i} \in \partial(H)$ converging to $u$ and $v_{i} \in \partial(H)$ converging to $v$. Then points $\sigma_{d}\left(u_{i}\right)$ and $\sigma_{d}\left(v_{i}\right)$ are on opposite sides of $\sigma_{d}(u)$. It follows that the leaf $\sigma_{d}(\ell)$ cuts the image of $H$, a contradiction with the assumption that $\mathcal{L}$ is a Thurston $d$-invariant lamination. Thus, $\operatorname{Bd}(G)$ consists of $2 n$ leaves, all of which map to $\sigma_{d}(\ell)$.

This implies that in the collection $\left\{\ell_{1}, \ldots, \ell_{d}\right\}=B$ there are exactly $n$ edges of $G$; denote their collection by $A$. Since $\mathcal{L}$ is clean, for each $k$ either $\ell_{k} \cap G=\emptyset$ or $\ell_{k} \subset \operatorname{Bd}(G)$. Hence there is a set $A^{\prime}$ of $d-n$ leaves in the collection $\ell_{1}, \ldots, \ell_{d}$ which are disjoint from $G$. Now, starting with $\ell$, select $n$ disjoint siblings of $\ell$ from $\operatorname{Bd}(G)$ and unite them with $A^{\prime}$ to get a full set of siblings of $\ell$. As this can be done for any $\ell$, we see that $\mathcal{L}$ is sibling $d$-invariant. 
Let $\mathcal{L}$ be a clean Thurston $d$-invariant lamination and let $\approx_{\mathcal{L}}$ be the equivalence relation defined in Definition 2.3 by Theorem 5.2 and Theorem $4.9 \approx_{\mathcal{L}}$ is a $d$ invariant laminational equivalence relation. By Corollary 4.10 and since $\mathcal{L}$ is clean, $\mathcal{L}$ is a q-lamination if and only if every chord in the boundary of the convex hull of an equivalence class of $\approx_{\mathcal{L}}$ is a leaf of $\mathcal{L}$. Below we further compare the laminations $\mathcal{L}$ and $\mathcal{L}_{\approx_{\mathcal{L}}}$ (Definition 2.4). For an equivalence class $\mathfrak{g}$, let $A_{\mathfrak{g}}$ be the union of all leaves of $\mathcal{L}$ which join points of $\mathfrak{g}$. Since $\mathcal{L}$ is clean, each $A_{\mathfrak{g}}$ is a point, a simple closed curve, a single leaf, or an arc containing at least two leaves. In all but the last case all leaves of $A_{\mathfrak{g}}$ are leaves of $\mathcal{L}_{\approx_{\mathcal{L}}}$. Thus, $\left[\mathcal{L} \backslash \mathcal{L}_{\approx_{\mathcal{L}}}\right] \cup\left[\mathcal{L}_{\approx_{\mathcal{L}}} \backslash \mathcal{L}\right]$ is contained in the countable union of the convex hulls of the equivalence classes $\mathfrak{g}_{i}$ for which $A_{\mathfrak{g}_{i}}$ is an arc with at least two leaves. We specify this in Corollary 5.3 .

Corollary 5.3. Let $\mathcal{L}$ be a clean Thurston d-invariant lamination and $\mathfrak{g}$ an equivalence class of $\approx_{\mathcal{L}}$ such that $A_{\mathfrak{g}}$ is an arc which contains at least two leaves of $\mathcal{L}$. Suppose that $\ell=\overline{a b} \subset \mathrm{CH}(\mathfrak{g})$. If $\ell \in \mathcal{L}_{\approx_{\mathcal{L}}} \backslash \mathcal{L}$, then there exists an infinite gap $U$ of $\mathcal{L}$ such that $\ell \backslash\{a, b\}$ is contained in the interior of $U$ and the subarc of $A_{\mathfrak{g}}$, which connects $a$ and $b$, is a maximal concatenation of leaves in $\operatorname{Bd}(U)$. On the other hand, if $\ell^{\prime}=\overline{u v} \in \mathcal{L} \backslash \mathcal{L}_{\approx_{\mathcal{L}}}$, then $\ell^{\prime} \backslash\{u, v\}$ is contained in the interior of $\mathrm{CH}(\mathfrak{g})$ and $\ell^{\prime}$ is the intersection of two infinite gaps of $\mathcal{L}$.

Proof. Suppose that $\ell=\overline{a b} \subset \mathrm{CH}(\mathfrak{g}), \ell \in \mathcal{L}_{\approx_{\mathcal{L}}} \backslash \mathcal{L}$. Clearly, $\ell$ cannot be approximated by leaves of $\mathcal{L}$. Moreover, since $\mathfrak{g}$ is finite, no leaf of $\mathcal{L}$ intersects $\ell$ inside $\mathbb{D}$. Hence there exists an infinite gap $U$ of $\mathcal{L}$ such that $\ell \backslash\{a, b\}$ is contained in the interior of $U$. Since $\mathcal{L}$ is clean, the subarc $[a, b]_{A_{\mathfrak{g}}}$ of $A_{\mathfrak{g}}$ is contained in the boundary of $U$. Moreover, since $\ell$ is an edge of $\mathrm{CH}(\mathfrak{g}),[a, b]_{A_{\mathfrak{g}}}$ is a maximal concatenation of leaves in $\operatorname{Bd}(U)$ (if it were not maximal, we could extend $\mathfrak{g}$ ).

Now, let $\ell=\overline{u v} \in \mathcal{L} \backslash \mathcal{L}_{\approx_{\mathcal{L}}}$. Then $\ell \backslash\{u, v\}$ is contained in the interior of $\mathrm{CH}(\mathfrak{g})$. Hence $\ell$ is isolated and there exist two gaps $U, V$ of $\mathcal{L}$ so that $\ell=U \cap V$. If one of these gaps is finite, then its boundary is a subset of $A_{\mathfrak{g}}$, a contradiction.

\section{Quadratic invariant Laminations}

In this section we study quadratic laminations. First we show that Corollary 4.10 can be made more precise in the quadratic case.

If a 2-invariant q-lamination $\mathcal{L}$ has a finite critical gap $L$, then one can insert a critical diameter connecting two vertices of $L$ and then pull it back along the backward orbit of $L$. Also, if $L$ has six vertices or more, one can insert a critical quadrilateral (with two edges which are edges of $L$ ) inside $L$ and then pull it back along the backward orbit of $L$. Such a quadrilateral can itself split into two triangles by a diameter and then can be pulled back along the backward orbit of $L$. In this way one can create proper sibling invariant laminations which are not q-laminations. If a lamination contains a finite critical polygon $L$ which contains a critical leaf (collapsing quadrilateral) in the interior of its convex hull, then we say that it has a critical splitting (by a leaf, or, resp., by a quadrilateral). Corollary 6.1 shows that these mechanisms are the only ways a proper quadratic lamination cannot be a q-lamination.

Corollary 6.1. A quadratic sibling invariant lamination is a q-lamination if and only if it is proper and does not have a critical splitting. 
Proof. Every q-lamination is proper and has no critical splitting. Now let $\mathcal{L}$ be a proper sibling invariant lamination without critical splitting. Define $\approx_{\mathcal{L}}$ and show that for each $\approx_{\mathcal{L}^{-}}$-class $\mathfrak{g}$ the edges of $\mathrm{CH}(\mathfrak{g})$ belong to $\mathcal{L}$. Suppose that for a $\approx_{\mathcal{L}^{-}}$ class $\mathfrak{g}$ there is an edge of $\mathrm{CH}(\mathfrak{g})$ not included in $\mathcal{L}$. By definition, there are finite concatenations of edges of $\mathcal{L}$ connecting all points of $\mathfrak{g}$. Hence $\mathrm{CH}(\mathfrak{g})$ cannot be a leaf and $\mathfrak{g}$ consists of more than two points. By Thurston's No Wandering Triangles Theorem Thu09] gaps of quadratic laminations are (pre)critical or (pre)periodic. Hence $\mathfrak{g}$ is either (pre)periodic or (pre)critical ( $\mathfrak{g}$ can first map into a critical class of $\approx_{\mathcal{L}}$ and then into a periodic class of $\approx_{\mathcal{L}}$, but not vice versa because $\mathcal{L}$ is proper).

Consider cases. Suppose that $\mathfrak{g}$ is (pre)periodic but not (pre)critical. Then for

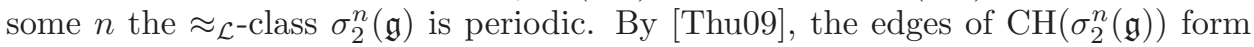
one periodic orbit of edges. Hence no diagonal of $\mathrm{CH}\left(\sigma_{2}^{n}(\mathfrak{g})\right)$ can be a leaf of $\mathcal{L}$ so that one edge of $\mathrm{CH}\left(\sigma_{2}^{n}(\mathfrak{g})\right)$ is a leaf of $\mathcal{L}$, and hence all edges of $\mathrm{CH}\left(\sigma_{2}^{n}(\mathfrak{g})\right)$ are leaves of $\mathcal{L}$. Since $\mathfrak{g}$ maps onto $\sigma_{2}^{n}(\mathfrak{g})$ one-to-one by our assumptions, then all edges of $\mathrm{CH}(\mathfrak{g})$ are in $\mathcal{L}$, as desired.

Now, suppose that $\mathfrak{g}$ is precritical and $\sigma_{2}^{n}(\mathfrak{g})$ is critical. Again, we may assume that $\mathrm{CH}(\mathfrak{g})$ is not a leaf. Since $\sigma_{2}^{n}(\mathfrak{g})$ is a critical $\approx_{\mathcal{L}}$-class, it must have $2 k$-edges and must map onto its image two-to-one. It follows that the edges of $\sigma_{2}^{n}(\mathfrak{g})$ are limits of sequences of $\approx_{\mathcal{L}}$-classes. Indeed, otherwise there are gaps of $\approx_{\mathcal{L}}$ sharing common edges with $\sigma_{2}^{n}(\mathfrak{g})$. By construction this would mean that these gaps are infinite, and hence a forward image of one of these gaps is a critical $\approx_{\mathcal{L}}$-class. Since we deal with quadratic laminations and $\mathfrak{g}$ is also critical, then this is impossible.

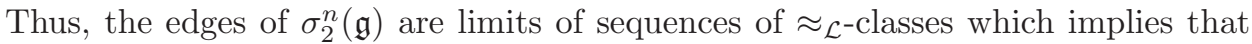
edges of $\sigma_{2}^{n}(\mathfrak{g})$ are leaves of $\mathcal{L}$. As before, since $\mathcal{L}$ is a Thurston invariant lamination, then all edges of $\mathfrak{g}$ are leaves of $\mathcal{L}$. Thus, in any case, if $\mathfrak{g}$ is a $\approx_{\mathcal{L}}$-class, then its edges are leaves of $\mathcal{L}$.

It remains to show that $\mathrm{CH}(\mathfrak{g})$ cannot contain any leaves of $\mathcal{L}$ in its interior. Indeed, suppose otherwise. We may assume that $\mathfrak{g}$ has at least 4 vertices. Suppose that $\mathfrak{g}$ is (pre)critical and $\sigma_{2}^{n}(\mathfrak{g})$ is critical. Let us show that any leaf inside $\sigma_{2}^{n}(\mathfrak{g})$ must have an image which is an edge or a vertex of $\sigma_{2}^{n+1}(\mathfrak{g})$. Indeed, it suffices to consider the case when $\sigma_{2}^{n}(\mathfrak{g})$ has at least six vertices. By the No Wandering Triangles Theorem [Thu09] for some $m \geq 0$, the gap $\sigma_{2}^{n+m}(\mathfrak{g})$ is periodic. By the above quoted result of Thu09, the edges of $\mathrm{CH}\left(\sigma_{2}^{n+m}(\mathfrak{g})\right)$ form one periodic orbit of edges. Hence if there is a leaf of $\mathcal{L}$ inside $\mathrm{CH}\left(\sigma_{2}^{n+m}(\mathfrak{g})\right)$, it will cross itself under the appropriate power of $\sigma_{2}$, a contradiction. Thus, any leaf inside $\sigma_{2}^{n}(\mathfrak{g})$ must have the image which is an edge or a vertex of $\sigma_{2}^{n+1}(\mathfrak{g})$.

We next show that such a leaf cannot exist. Since $\mathcal{L}$ does not admit a critical splitting, we also need to show that no other splitting of $\mathrm{CH}\left(\sigma_{2}^{n}(\mathfrak{g})\right)$ by leaves of $\mathcal{L}$ is possible. Indeed, suppose that there are leaves of $\mathcal{L}$ inside $\mathrm{CH}\left(\sigma_{2}^{n}(\mathfrak{g})\right)$. Since $\mathcal{L}$ does not have critical splitting, it cannot be just one critical leaf, nor can it be a quadrilateral or a quadrilateral with a critical leaf inside. Now, suppose that there is a unique leaf $\ell$ of $\mathcal{L}$ inside $\sigma_{2}^{n}(\mathfrak{g})$ such that $\sigma_{2}(\ell)$ is an edge of $\sigma_{2}^{n+1}(\mathfrak{g})$. Then it has to have a sibling leaf which will also be a leaf inside $\sigma_{2}^{n}(\mathfrak{g})$. Hence $\sigma_{2}^{n}(\mathfrak{g})$ contains a collapsing quadrilateral, a contradiction. As these possibilities exhaust all possibilities for leaves inside $\mathrm{CH}\left(\sigma_{2}^{n}(\mathfrak{g})\right)$, it follows that there are no leaves inside $\mathrm{CH}\left(\sigma_{2}^{n}(\mathfrak{g})\right)$ and hence no leaves inside $\mathrm{CH}(\mathfrak{g})$, as desired.

Corollary 6.2. Suppose that $\mathcal{L}$ is a clean Thurston 2-invariant lamination. Then $\mathcal{L}$ is a q-lamination. 
Proof. Suppose that $\mathcal{L}$ is a clean, Thurston 2-invariant lamination. By Theorem 5.2 . $\mathcal{L}$ is proper and sibling invariant. Moreover, since $\mathcal{L}$ is clean, it does not have critical splitting. Hence the result follows from Corollary 6.1.

\section{ACKNOWLEDGMENTS}

The authors would like to thank the referee for useful suggestions and comments.

\section{REFERENCES}

[BCO08] A. Blokh, C. Curry, and L. Oversteegen, Locally connected models for Julia sets, Advances in Mathematics, 226 (2011), 1621-1661. MR.2737795 (2012d:37106)

[BL02] A. Blokh and G. Levin, Growing trees, laminations and the dynamics on the Julia set, Ergodic Theory and Dynamical Systems, 22 (2002), 63-97. MR.1889565 (2003i:37045)

[BO06] A. Blokh and L. Oversteegen, The Julia sets of quadratic Cremer polynomials, Topology and its Applications, 153 (2006), 3038-3050. MR.2248408 (2007j:37070)

[Chi07] D. Childers, Wandering polygons and recurrent critical leaves, Ergodic Theory and Dynamical Systems, 27 (2007), 87-107. MR2297088(2008b:37077)

[Dou93] A. Douady, Descriptions of compact sets in $\mathbb{C}$, Topological Methods in Modern Mathematics, Publish or Perish (1993), 429-465. MR1215973 (94g:58185)

[Kiw02] J. Kiwi, Wandering orbit portraits, Transactions of the American Mathematical Society, 354 (2002), 1473-1485. MR.1873015 (2002h:37070)

[Kiw04] J. Kiwi, Real laminations and the topological dynamics of complex polynomials, Advances in Mathematics, 184 (2004), 207-267. MR2054016(2005b:37094)

[Mim10] D. Mimbs, Laminations: A topological approach, Ph.D. thesis, University of Alabama at Birmingham, 2010. MR2801683

[Nad92] S. Nadler, Continuum Theory: An Introduction, Marcel Dekker, NY, 1992. MR.1192552 (93m:54002)

[Thu09] W. Thurston, Polynomial dynamics from Combinatorics to Topology, 1-109 in Complex Dynamics: Families and Friends, ed. Dierk Schleicher, A K Peters, Wellesley, MA, 2009. MR1500163 (2010a:37001)

Department of Mathematics, University of Alabama at Birmingham, Birmingham, AlABAMA 35294-1170

E-mail address: ablokh@math.uab.edu

Department of Mathematics, University of Alabama at Birmingham, Birmingham, AlABAMA 35294-1170

Current address: Department of Mathematics, Lee University, Cleveland, Tennessee 373203450

E-mail address: dmimbs@leeuniversity.edu

Department of Mathematics, University of Alabama at Birmingham, Birmingham, AlABAMA 35294-1170

E-mail address: overstee@math.uab.edu

Faculteit der Exacte Wetenschappen, Afdeling Wiskunde, Vrije Universiteit, De Boelelaan 1081A, 1081 HV Amsterdam, The Netherlands

E-mail address: kirstenvalkenburg@gmail.com 